\title{
Prediction of Gas Permeability of Block-Segregated Polymeric Membranes by an Effective Medium Model.
}

\author{
AlbertoTena $^{1,3}$, Mónica de la Viuda ${ }^{1}$, Laura Palacio ${ }^{1,2}$, Pedro Prádanos ${ }^{1,2}$, Ángel \\ Marcos-Fernández ${ }^{1,3}$, Ángel E. Lozano ${ }^{1,3}$ and Antonio Hernández ${ }^{1,2}$ \\ ${ }^{1}$ Smap UA-UVA-CSIC, Parque Científico Universidad de Valladolid, Paseo de Belén, s/n, 47011 \\ Valladolid, Spain. \\ ${ }^{2}$ Dept. Física Aplicada, Facultad de Ciencias, Universidad de Valladolid, Paseo de Belén, 7, 47011 \\ Valladolid, Spain \\ ${ }^{3}$ Instituto de Ciencia y Tecnología de Polímeros, CSIC, Juan de la Cierva 3, 28006 Madrid, Spain.
}

\section{Summary}

A complete series of aliphatic aromatic copoly(ether-imide)s, based on aromatic dianhydrides (BPDA, $B K D A$ or PMDA) and mixtures of an aromatic diamine (ODA) and an aliphatic diamine terminated poly(ethylene oxide) PEO(2000) (Mw=2000 $\mathrm{g} / \mathrm{mol})$ or PEO(6000) (Mw=6000 $\mathrm{g} / \mathrm{mol})$, has been synthesized using different PEO contents. Cast films of these copolymers have been thermally treated to ensure the segregation of the linear PEO chains from the aromatic portion of these copoly(ether-imide)s.

Gas permeability $\left(\mathrm{O}_{2}, \mathrm{~N}_{2}, \mathrm{CO}_{2}\right.$ and $\left.\mathrm{CH}_{4}\right)$ of membranes, made from these copolymers with different proportions of PEO, were compared with the predictions of various electrical or thermal conductivity models adapted to gas permeability. Several of these models, from Maxwell-Garnett to percolation theory, proved to be inaccurate. Nevertheless, the model based on the Effective Medium Approximation (EMA) is proved here to succeed in predicting the main features of the experimental results for all mixture proportions, taking as the starting point the sole input of pure homopolymer permeabilities. Specifically, this later method was able to calculate the volume fraction for the maximum increase of permeability, a common feature for all the studied segregated copolymer membranes. The model was even able to predict the permeabilities of a three phase system consisting in the aromatic (BKDA-ODA) phase plus a mixture of amorphous PEO(6000) and crystalline PEO(6000).

Keywords: Copoly(ether-imide) membrane; Phase segregation; Thermal treatment; Gas separation; Effective Medium Approximation, Percolation, Maxwell-Garnett Models.

\section{*membrana@termo.uva.es}

Tel.: $\quad 34-983423134$

Fax.: 34-983423013 


\section{Introduction}

Nowadays, the role of polymeric membranes applied to gas separation is being more and more important. Although some of these materials have been used in industrial separations[1], it is well known that a lot of research is still necessary to discover or refine materials having an equilibrium of properties making them suitable to be employed in applications demanded nowadays by the industry. In all cases, an adequate balance of high permeability and good selectivity must be achieved [2-3].

In order to be useful in gas separation applications where one or several of the gases to separate-be separated is a condensable gas such as $\mathrm{CO}_{2}$ or $\mathrm{CH}_{4}$, the polymeric membrane should show a preferential affinity for them. In addition, these membranes must have good mechanical and thermal resistance.

Glassy polymers and in particular polyimides are well known by their excellent thermal oxidative stability, good organic solvent resistance and exceptional mechanical properties, along with an extraordinary ability to separate complex mixtures of gases in diverse applications [4-6]. Thus, it has been widely recognized that the use of aromatic polyimides is one of the best alternatives to be chosen among all the polymeric membranes for gas separation applications [7].

Typically, these materials have good selectivity and not very high permeability [8-9]. It could therefore be interesting to increase the affinity of the material matrix for condensable gases such as $\mathrm{CO}_{2}$, or $\mathrm{CH}_{4}$ as one of the most effective approaches.

Aromatic-aliphatic block-copolymers usually combine a hard block and a soft block. The hard block can be formed by a polymer with well-packed and highly rigid structures; as a result it forms a glassy phase with usually low free volume. In contrast, the soft block can consist in a polymer with more flexible, low $\mathrm{T}_{\mathrm{g}}$, chains, which can form a rubbery phase having high free volume. Also, when aromatic-aliphatic block copolymers are phase-separated, for instance, by an adequate thermal treatment, their glassy polymer parts could provide mechanical support. The rubbery segments, due to the nature of the flexible chain structure, should allow an efficient transport of gas, 
giving a good permeability to the material [10-11]. Among all the block copolymers,

The synthesis of new PEO systems using different aromatic diamines showed that an increase of rigidity in the hard part promoted the phase segregation process [12]. When the temperature of treatment of the films increased, phase segregation improved [13]. Analogous trends have been proved when diverse types of aromatic dianhydrides of different rigidity were employed [14]. These processes depended also on the type of polyether used, because the phase segregation and the final properties of the membranes can also vary depending on the nature of the soft part [15].

Mechanical properties of the membranes tended to worsen when the amount of polyether increased, because the amount of the hard part (which provides the mechanical strength of the final polymer) decreased. At the same time, the segregation and the permeation properties improved [16-17, 18].

Polyethers such as PEO can experience easy crystallization processes when the molecular weight exceeds a certain value [16]. In gas separation this increase of crystallinity is undesirable because ordered structures have low or null permeation capacity. When PEO is in amorphous state, it can be said that the permeability increases with the length of the aliphatic part, mainly due to the improvement in phase segregation [16]. It is clearly important to find a good balance between the hard and the soft block segments in order to provide good permeability without having a dramatic loss in mechanical properties.

Many composite systems, as for example mixed matrix membranes, MMM, have been modeled to correlate the overall permeability with those of the component phases by applying Maxwell-Garnett, MG, based treatments. Herein, we have applied this treatment to phase segregated membranes and we have found difficulties to model, within this theoretical frame, the range of intermediate concentrations. In this paper it is demonstrated that more symmetric schemes as the effective medium approximations, EMA, reproduce well enough the composition ranges where none of the phases, forming the material, can be considered as dispersed in a continuous matrix. 


\section{Experimental}

The segregated copoly(etherimide)s whose permeabilities are modeled here have been synthetized, thermally segregated and characterized previously by our group [12, 15-16, 19]. A brief summary of these aspects is provided below.

\subsection{Chemicals}

3,3',4,4'- Biphenyltetracarboxylic dianhydride (BPDA); 3,3',4,4'-benzophenone tetracarboxylic dianhydride (BTDA or BKDA); pyromellite dianhydride (PMDA), and 4,4'-oxydianiline (ODA) were purchased from Aldrich. These products were purified by sublimation at high vacuum just before use. Polyoxyethylene bis(amine) (Jeffamine ED2003, $n=41$ ) with nominal molecular weight of $2000 \mathrm{~g} / \mathrm{mol}$, was kindly donated given by Huntsman ${ }^{\circledR}$ (Holland) (PEO-2000 from here on). $\alpha, \omega$-Diamine-poly(ethylene oxide) with nominal molecular weight of $6000 \mathrm{~g} / \mathrm{mol}$, were obtained from Kawaken Fine Chemicals Co., Ltd. (Tokyo, Japan), (PEO-6000 from here on). These polyethers were dried at $70{ }^{\circ} \mathrm{C}$ in vacuum for 5 hours and stored in a desiccator at vacuum until use. Anhydrous N-methylpyrrolidinone (NMP), used as the polymerization solvent, was purchased from Sigma-Aldrich Co.

\subsection{Synthesis of copoly(ether-imide)s}

The samples were synthesized by combination of a dianhydride (BPDA, BKDA or PMDA) with mixtures of an aromatic diamine (ODA), and diverse proportions of an aliphatic diamine (PEO). The corresponding copoly(ether-imide)s will be designated by adding $\mathrm{cPI}$ to the $\mathrm{w} / \mathrm{w}$ percentage of the aliphatic proportion.

Diamine-terminated poly(oxyethylene oxide $)-\operatorname{PEO}(2000)$ or $\mathrm{PEO}(6000)-(x \mathrm{mmol})$, and 4,4'-oxydianiline (ODA) ( $y \mathrm{mmol}$ ) in several weight ratios were dissolved in anhydrous NMP $(5 \mathrm{mmol}(x+y) / 10 \mathrm{~mL})$ in a $100 \mathrm{~mL}$ three-necked flask blanketed with nitrogen. 
Then, the reaction mixture was cooled down to $0{ }^{\circ} \mathrm{C}$, and under mechanical stirring, a maintained overnight at room temperature. During this time the dianhydride was completely dissolved and the solution reached high viscosity.

In Figure 1 the corresponding structures of the aromatic and aliphatic homopolymers corresponding to the copoly(ether-imide)s synthetized are shown along with their densities

\section{Figure 1}

\subsection{Preparation of the copolyimide dense films.}

The resultant viscous copolyamic acid solution was diluted with NMP to the appropriate viscosity for casting, filtered through a nominal \#1 fritted glass funnel, degassed, and cast onto a leveled glass plate. The resulting film was covered with a conical funnel to avoid fast evaporation of the solvent, dried at $80{ }^{\circ} \mathrm{C}$ overnight, and finally treated at several temperatures from 160 to $250^{\circ} \mathrm{C}\left(275^{\circ} \mathrm{C}\right.$ for the BKDA containing films) for 6 hours until $200^{\circ} \mathrm{C}$ and 30 min over this temperature in a vacuum oven, in order to achieve a complete imidization. Films of copolymers having 50-70 $\mu \mathrm{m}$ in thickness were obtained. After that, thermal treatments under an inert atmosphere were carried out at different temperatures.

\subsection{Characterization of the membranes.}

All the copoly(eherimide)s studied here were treated at different temperatures (as mentioned above) and the resulting films extensively characterized [12-19]. They were and-Phase segregation was confirmed by Small-Angle X-ray Scattering (SAXS) observing that this process increases with temperature. Glass transition temperatures, crystallinity and mechanical properties were obtained by using Differential Scanning Calorimetry (DSC), Thermo Gravimetric Analysis (TGA), Thermo Mechanical Analysis (TMA) and Dynamic Mechanical Analysis (DMA). All the films showed good mechanical properties. 
Permselectivities were obtained by using "time lag" permeators at 3 bar and $30{ }^{\circ} \mathrm{C}$ (some measurements were done at $50{ }^{\circ} \mathrm{C}$ when crystallinity was present in the case of PEO-6000).

\section{Theory}

The membranes composed by two or more different phases should have permeability values placed between those for pure phases. Copolyimides consisting in an aromatic polyimide part (hard segments) and an aliphatic polyether one (soft PEO chains) should be modeled as a system formed by two intermixed phases.

It has been generally $[16,20,21]$ found that for intermediate volume fractions there is a range where none of the Maxwell-Garnett predictions are accurate. It has been also noticed that low PEO proportions are well predicted by the Maxwell-Garnett model assuming that PEO is the dispersed phase although for high PEO proportions the Maxwell-Garnett model with PEO as the continuous phase fits better the experimental results [20]. Of course there is a range where neither the PEO rich nor the aromatic rich phases can be taken as disperse or (discontinuous). This intermediate range has resisted all attempts to be predictable or even understood [19]. Among the attempts made we can mention the addition of an ad hoc parameter, so called "chain immobilization factor", that should be evaluated as a function of composition to take into account the differences between the predictions of Maxwell-Garnett model for small fractions of one of the phases and the experimental results [21]. Other possibility is to patch both the Maxwell-Garnet predictions corresponding to PEO as disperse and to PEO as continuous phase by introducing a threshold value for the composition that again has to be adapted "ad hoc" to the experimental results [19].

A revision will be made here of some of the main models that can be used to predict permeabilities for different proportions of a multiphase gas separation membrane. This revision cannot be exhaustive as far as there are a huge plethora of models more or less similar to each other which were originally proposed for electric or thermal conductivity or for mechanical properties [22-24]. The models will, of course, be adapted to gas 
permeation attending to the well-known analogy existent between the transport processes [25].

\subsection{Maxwell-Garnett Models}

The Maxwell-Garnett approximation, [26-27] also known as the Clausius-Mossotti approximation, is one of the most widely used methods for calculating the bulk dielectric properties of inhomogeneous materials [28-29]. It is useful when one of the components can be considered as a host in which inclusions of the other component or components are embedded. It involves an exact calculation of the field induced in the uniform host by a single spherical or ellipsoidal inclusion and an approximate treatment of its distortion by the electrostatic interaction between the different inclusions. This distortion is caused by the charge dipoles and higher multipoles induced in the other inclusions. The induced dipole moments cause the longest range distortions and their average effect is included in the Maxwell approximation which results in a uniform field inside all the inclusions.

These approaches have been extensively used to study the properties of two-component mixtures in which both, the host and the inclusions, are isotropic materials with scalar dielectric coefficients. Nevertheless, applying relaying inthe known analogies of transport phenomena, this theoretical frame has been used to model: electrical conductivity, thermal conductivity and elasticity parameters. They have been also extensively used for permeation modeling.

For gas separation processes, it has been demonstrated that systems consisting in a disperse phase embedded in a continuous matrix can be described in terms of these models that have been called Maxwell-Garnett or Maxwell-Eucken models. Within the frame of these theoretical schemes, the effective permeability is:

$$
P_{e f f}=P_{c}\left[\frac{P_{d}+2 P_{c}-2\left(P_{c}-P_{d}\right)}{P_{d}+2 P_{c}+\left(P_{c}-P_{d}\right)}\right]
$$


Where $P_{\text {eff }}$ is the effective permeability, $P_{c}$ and $P_{d}$ are the permeabilities of the continuous and disperse phase, respectively, and $\phi_{\mathrm{d}}$ is the volume fraction of the dispersed phase in the block copolymer.

In our study, of course the continuous phase can be the aromatic or the aliphatic rich portions depending on the composition of the copolymer. For this reason, it should be necessary to make two different predictions depending on which segment is taken as the continuous phase or the disperse one. This is necessary because Equation (1) is not symmetric and if the roles of the filler and the continuous phase are swapped, different results are obtained.

Bruggeman [30] introduced a popular approximation that is now widely known as the differential scheme or the Bruggeman equation. Bruggeman proceeds from the premise that the fields of neighboring particles can be taken into account by adding the dispersed particles incrementally, taking the surrounding medium to be the existing composite at each stage.

Translated to gas permeability, the Bruggeman equation says that the effective permeability of an inhomogeneous medium is given by:

$\frac{P_{d}-P_{e f f}}{P_{d}-P_{c}}\left(\frac{P_{c}}{P_{\text {eff }}}\right)^{1 / d}=1-\phi_{d}$

where $\mathrm{d}=3$ for spherical fillers [31].

The Bruggeman equation is appropriate for composites containing spherical particles with a very wide size distribution. Ideally, the size distribution should be wide enough so that any two spheres of comparable size are far from each other. Note that Equation (2) needs to be solved numerically and it is also asymmetric, in the sense commented when referring to Equation (1), i.e. it is not single valued.

Some models have been applied without any pursued symmetry between filler and continuous matrix because they are intended for low content of filler within a continuous matrix. Most of these models lead to divergences in permeability for filler contents over a certain value. They belong to this class, for example, the models due to 
Bruggeman [30], Bötcher [32] and De Loor [33] and corresponding to Equations (3) to (5) [34]:

$$
\begin{aligned}
& P_{e f f}=P_{c}\left(1-\phi_{d}\right)^{-3} \\
& P_{e f f}=P_{c}\left(1-\phi_{d}\right)^{-1} \\
& P_{e f f}=P_{c}\left(1-\phi_{d}\right)\left(1-2 \phi_{d}\right)^{-1}
\end{aligned}
$$

The Lewis and Nielsen model [35-37], originally proposed for the elastic modulus of particulate composites, gives:

$$
\begin{aligned}
& P_{e f f}=P_{c}\left[\frac{1+A \phi_{d} \frac{\left(P_{d} / P_{c}\right)-1}{\left(P_{d} / P_{c}\right)+A}}{1-\psi \phi_{d} \frac{\left(P_{d} / P_{c}\right)-1}{\left(P_{d} / P_{c}\right)+A}}\right] \\
& \psi=1+\phi_{d}\left(\frac{1-\bar{\phi}_{d}}{\bar{\phi}_{d}^{2}}\right)
\end{aligned}
$$

The values of A and $\bar{\phi}_{d}$ were given for many geometric shapes and orientations [38-40]. A is 2 for spheres in a unidirectional transport, $\bar{\phi}_{d}$ is 0.64 for random close packing of uniform spheres. $\bar{\phi}_{d}$ is the maximum $\phi_{d}$ that is the maximum packing volume fraction for a given inclusion size and shape.

The Pal model [41] assumes that:

$$
\frac{P_{d}-P_{e f f}}{P_{d}-P_{c}}\left(\frac{P_{c}}{P_{e f f}}\right)^{1 / d}=\left(1-\frac{\phi_{d}}{\bar{\phi}_{d}}\right)^{\bar{\phi}_{d}}
$$

where $d=3$ as in Equation (2) for spheres like for the original work of Bruggeman (Equation (2)). Pal originally proposed this expression for the thermal conductivity of particulate composites using a differential scheme and taking into account the packing difficulty of particles and modifying the Bruggeman model.

Equations (6) to (8) belong also to the divergent or low filler fraction models. 


\subsection{Effective Medium Theory}

Actually, when both phases are not continuous and there is a random dispersion of them, the Maxwell-Garnett approach is not reasonable. Within the frame of the Maxwell-Garnett's model, the continuous and discontinuous phases must be first identified because Equations (1) to (8) are not symmetric as mentioned. Systems where the formation of an internal network in the structure is observed are more accurately represented with the effective medium theory (EMT) that treats the contributions of each phase equally. As the equation of Maxwell-Garnett, EMT is derived from the solution of the Laplace equation applied to a single sphere surrounded by a continuous medium, and subjected to a steady-state concentration gradient around the spheres. Maxwell-Garnett assumed that the local distortions to the concentration distributions around the dispersed spheres did not affect their neighbors. The essence of EMT, however, lies in the assumption that for a completely random distribution of components, the effect of local distortions to the concentration distribution caused by individual inclusions could be averaged in such way that over a sufficiently large volume (or ensemble) the concentration distribution within the material could be approximated by a material having an uniform concentration distribution and permeability $\mathrm{P}_{\text {eff }}$ [42-43].

Effective medium approximations or effective medium theory (sometimes abbreviated as EMA or EMT) are physical models that describe the macroscopic properties of composite materials, which are developed from averaging the multiple values of the constituents that directly make up the composite material. At the constituent level, the values of the materials vary and are inhomogeneous. Precise calculation of the many constituent values is nearly impossible. The EMA methodologies are based [30] on a self-consistent procedure in which a grain of one of the components is assumed to have a convenient shape (usually spherical or ellipsoidal) and to be embedded in an effective medium whose properties are determined self-consistently [29, 43]. EMA treats equally both the components as shown in its fundamental equation, [44, 45]:

$\phi_{d} \frac{P_{d}-P_{e f f}}{P_{d}+2 P_{e f f}}+\phi_{c} \frac{P_{c}-P_{e f f}}{P_{c}+2 P_{e f f}}=0$ 
Note that this frame can be extended to $n$ phases by using:

$\sum_{i=1}^{n} \phi_{i} \frac{P_{i}-P_{e f f}}{P_{i}+2 P_{e f f}}=0$

Although the distinction of dispersed and continuous phases losses its meaning as far as the two phases are treated in the same way and the roles of both the phases could be interchanged and the predicted overall permeability would not change, we will continue naming disperse phase to the PEO domains and continuous phase to the aromatic rich one. Equation (9) can be solved to:

$$
P_{e f f}=\frac{1}{4}\left(\gamma+\sqrt{\gamma^{2}+8 P_{d} P_{c}}\right)
$$

with

$\gamma=\left(3 \phi_{d}-1\right) P_{d}+\left(3 \phi_{c}-1\right) P_{c}$

of course using the identity $\phi_{c}=1-\phi_{d}$. All these EMA calculations are particularly appropriate for composites and polycrystals in which the grains of the various components are randomly and symmetrically distributed, so that none of the components is identifiable as a host in which the others are preferentially embedded.

Because of the asymmetry of the assumed geometry, and the approximation, the results are likely to differ somewhat from the unevaluated (symmetric) EMA. This geometrical distinction may be easily understood by noting that the EMA and MG approximations are exact for two different microgeometries [28]. The EMA becomes exact in a hierarchical geometry where the two components play symmetrical geometric roles [46]. In contrast, the MG approach is exact for a geometry where the entire space is filled with equal spheres, each with identical ratio, such that one component is the core material and the other is the coating material (the host).

\subsection{Modifications of the Maxwell-Garnett models}

Modifications of the Maxwell-Garnett model have been done in two main directions. Firstly, the original asymmetric model has been modified to be symmetrical. And also the model has been modified to take into account the appearance of different phases 
corresponding, for example, to the interfaces between the matrix and the disperse phases or to take into account the differences of: size, isotropy etc. In this work, we will only mention in some detail the modifications addressed to make the approach symmetric and to take into account the possible presence of more phases.

The Levy model was originally proposed as a purely mathematical averaging of the Maxwell-Eucken model with each of the two phases being treated as continuous [47]. This model was recently given a physical interpretation by Wang et al. [48]. In terms of gas permeability the model states that:

$$
\begin{aligned}
& P_{e f f}=P_{c}\left[\frac{P_{d}+2 P_{c}-2\left(P_{c}-P_{d}\right) F}{P_{d}+2 P_{c}+\left(P_{c}-P_{d}\right) F}\right] \\
& F=\frac{(2 / g)-1+2 \phi_{d}-\sqrt{\left[(2 / g)-1+2 \phi_{d}\right]^{2}-\left(8 \phi_{d} / g\right)}}{2} \\
& g=\frac{\left(P_{c}-P_{d}\right)^{2}}{\left(P_{d}+P_{c}\right)^{2}+\frac{P_{d} P_{c}}{2}}
\end{aligned}
$$

which is symmetrical and single valued although, as we will see below, its symmetry could be inadequate in some cases.

In order to treat three-phase-systems the Maxwell-Garnett model has been modified as:

$$
P_{e f f}=P_{c}\left[\frac{P_{d}+2 P_{c}-2\left(P_{c}-P_{d}\right) F}{P_{d}+2 P_{c}+\left(P_{c}-P_{d}\right) F}\right]=P_{c}\left[\frac{2\left(1-\phi_{d}\right)+\left(1+2 \phi_{d}\right)\left(P_{d} / P_{c}\right)}{\left(2+\phi_{d}\right)+\left(1-\phi_{d}\right)\left(P_{d} / P_{c}\right)}\right]
$$

with

$$
P_{d}=P_{i n}\left[\frac{2\left(1-\phi_{k}\right)+\left(1+2 \phi_{k}\right)\left(P_{k} / P_{i n}\right)}{\left(2+\phi_{k}\right)+\left(1-\phi_{k}\right)\left(P_{k} / P_{i n}\right)}\right]
$$

$P_{\text {eff }}$ is the permeability of the ensemble continuous phase plus disperse phase, $P_{d}$ is the permeability of a single core plus interfacial shield particle and $\mathrm{P}_{\text {in }}$ is the permeability of the interfacial shield covering the particle core that has a permeability $\mathrm{P}_{\mathrm{k}} . \phi_{\mathrm{d}}$ is the 
volume fraction of inclusions and $\phi_{\mathrm{k}}$ is the fraction of the core in the core+shield system. This model has been developed to take into account the existence of such shields covering inclusions in mixed matrix membranes [49-51].

The Felske model [52] has been proposed for such complex (three phases) inclusions. This model gives:

$$
\begin{aligned}
& P_{e f f}=P_{c}\left[\frac{2\left(1-\phi_{d}\right)+\left(1+2 \phi_{d}\right)(\alpha / \beta)}{\left(2+\phi_{d}\right)+\left(1-\phi_{d}\right)(\alpha / \beta)}\right] \\
& \alpha=\frac{\left(2+\delta^{3}\right) P_{k}-2\left(1-\delta^{3}\right) P_{i n}}{P_{c}} \\
& \beta=1+2 \delta^{3}-\left(1-\delta^{3}\right) \frac{P_{k}}{P_{i n}}
\end{aligned}
$$

And $\delta$ is the ratio of outer-shield to core radii.

The Felske model gives almost the same results than the modified Maxwell-Garnett model and although it is somewhat simpler, it has the same limitations than the original Maxwell-Garnett model [53].

As done by Lewis and Nielsen, $\bar{\phi}_{d}$ can be introduced in the Felske model to give [36, 53]:

$P_{\text {eff }}=P_{c}\left[\frac{1+2 \frac{\alpha-\beta}{\alpha+2 \beta} \phi_{d}}{1+\frac{\alpha-\beta}{\alpha+2 \beta} \psi \phi_{d}}\right]$

with $\psi$ given by Equation (7).

\subsection{Percolation Models}

The original problem was proposed [54] for the fluid flow in a porous medium. It has been applied to many other related problems including many phenomena in physics, chemistry and materials science [55]. 
A two phase system formed by inclusions dispersed in a continuous matrix can be modeled by a (3D) lattice in which each site can be occupied or empty with certain complimentary probabilities. In mathematics, percolation theory describes the behavior of connected clusters in such a random graph. In terms of gas permeability, an occupied site has a permeability $P_{d}$ while an unoccupied site has a permeability $P_{c}$. The fundamental premise of percolation theory is the appearance of a sharp increase in the effective permeability of the disordered media at a critical volume fraction known as the percolation threshold $\phi_{\mathrm{t}}$ at which long-range connectivity of the system appears.

When $P_{d} \neq 0, \mathrm{P}_{\mathrm{c}}=0$, and $\phi_{\mathrm{d}}<\phi_{\mathrm{t}}$, no macroscopic conducting pathway exists and no permeation happens through the composite phase. When $\phi_{\mathrm{d}}>\phi_{\mathrm{t}}$, however, the system becomes permeable as a connected path appear through the composite material. The existence of a critical percolation threshold for electrical conductivity has been demonstrated for a wide variety of fillers, all at concentrations below the maximum packing fraction, $\bar{\phi}_{d}$ [56]. The percolation threshold in an actual composite material is in general a function of the lattice structure of the phases, and ranges from $\phi_{t} \approx 0.2$ for a face-centered cubic arrangement to $\phi_{t} \approx 0.7$ for a honeycomb arrangement and it can be exactly calculated for certain simple lattices $[44,45]$.

Electrical conductivity has been treated frequently within percolation models [57] but also thermal conductivity has been modeled as percolative [58-59]. However, many researchers have reported that no percolation appears in thermal conductivity [60]. It may be noted that in contrast to the electrical conductivity, the concentration dependence of thermal conductivity does not show any swift leap that could be interpreted as a percolation threshold. This is because thermal conductivity of the dispersed filler is usually comparable to that of the polymer matrix. Given that this is even more certain for the gas permeabilities through disperse and continuous phases in a composite, we could find difficult to justify the appearance of percolation in the systems to be studied here. Nevertheless, as will be seen below, there is actually a sudden increase in the effective permeability of our two phase systems, thus we will adapt and try to use the most promising percolation model proposed for the thermal conductivity. 
This model was proposed by Zhang et al. [61-62] and translated to permeability says that:

$P_{e f f}=P_{d}\left(\frac{P_{t}}{P_{d}}\right)^{\left[\frac{1-\phi_{d}}{1-\phi_{t}}\right]^{N}}$

$\mathrm{P}_{\mathrm{t}}$ is the permeability at the threshold (percolation) composition given by $\phi_{\mathrm{t}}$ and $\mathrm{N}$ is the percolation exponent. This model contains actually 3 parameters to be fitted. Although the values of $\mathrm{P}_{\mathrm{t}}$ and $\phi_{\mathrm{t}}$ can be obtained by an analysis of the experimental data, $\mathrm{n}$ has to be fitted or evaluated for some fixed point.

\section{Results and Discussion}

In Figure 2 the $\mathrm{CO}_{2}$ permeability of the copolyimide BPDA-ODA-PEO(2000) thermally treated to $250{ }^{\circ} \mathrm{C}$ as a function of the volume fraction of PEO $\left(\phi_{d}\right)$ is shown. The predictions of Equations: (1) (Maxwell-Garnett); (2) (Brugeman); (9), (11) and (12) (EMA) and of Equations (13), (14) and (15) (Levy) are shown. It seems clear that the EMA model reproduces well all the main features of experimental data. It seems clear that the presence of an inflexion point in the experimental data is only predicted by the EMA and the Levy models. The vertical double line in Figure 2 corresponds to the volume fraction of PEO for this inflexion point.

\section{Figure 2}

When Equations (11) and (12) are taken into account it is seen that an inflexion point always appears, within the EMA model when $\log \mathrm{P}_{\mathrm{eff}}$ is plotted versus $\phi_{\mathrm{d}}$. This inflexion point can be calculated by solving $\mathrm{d}^{2} \log \left(\mathrm{P}_{\mathrm{eff}}\right) / \mathrm{d}_{\mathrm{d}}{ }^{2}=0$ and corresponds to the volume fraction of the disperse phase at which $\mathrm{P}_{\text {eff }}$ increases at its maximum pace. This happens when:

$\hat{\phi}_{d}=\frac{1-2 \lambda}{3(1-\lambda)}$ or for all $\phi_{\mathrm{d}}$ when $\lambda=1$

Here

$\lambda=\frac{P_{d}}{P_{c}}$

59 
It is worth remembering that the roles of continuous and disperse phases could be interchanged.

The behavior of the function given in Equation (23) is shown in Figure 3. Note that the maximum increase of $\mathrm{P}_{\mathrm{eff}}$ happens for $\hat{\phi}_{d} \approx 1 / 3$ if $\mathrm{P}_{\mathrm{d}}>>\mathrm{P}_{\mathrm{c}}\left(\right.$ or $\hat{\phi}_{d} \approx 2 / 3$ if $\mathrm{P}_{\mathrm{c}}>>\mathrm{P}_{\mathrm{d}}$ ). Moreover only when $\mathrm{P}_{\mathrm{d}}=\mathrm{P}_{c} / 2$ or $\mathrm{P}_{c}=\mathrm{P}_{\mathrm{d}} / 2$ there is a maximum increase of $\mathrm{P}_{\text {eff }}$ at $\hat{\phi}_{d}=0$ or $\hat{\phi}_{d}=1$. In any case, this inflexion point cannot appear within the range $1 / 3<\hat{\phi}_{d}<2 / 3$. Note that according to the Levy model this inflexion would appear at $\hat{\phi}_{d}=1 / 2$. This is the justification to our attribution of an excessive symmetry to this model that, nevertheless, is the only model along with the EMA model to be able to predict an inflexion point.

Figure 3

In Figure 4 the $\mathrm{O}_{2}, \mathrm{~N}_{2}$ and $\mathrm{CH}_{4}$ permeabilities of the copolyimide BPDA-ODA$\operatorname{PEO}(2000)$ thermally treated at $250^{\circ} \mathrm{C}$ as a function of the volume fraction of PEO $\left(\phi_{d}\right)$ are depicted. The same features as seen in Figure 2 are now observed and in this case only the predictions of the EMA model are shown.

\section{Figure 4}

In Figure 5 the permeability for $\mathrm{O}_{2}$ of the copolyimide BPDA-ODA-PEO(2000) thermally treated at $250{ }^{\circ} \mathrm{C}$ is shown as a function of the volume fraction of PEO $\left(\phi_{\mathrm{d}}\right)$ along with the predictions of the models given by Equations: (3) (Brugeman) ; (4) (Bötcher); (5) (De Loor); (6) and (7) (Lewis-Nielsen) and by Equation (8) (Pal). The EMA model predictions, already shown in Figure 4 are drawn too for the sake of comparison. It seems clear that all these models diverge or at least do not approximate to the pure PEO permeability for $\phi_{\mathrm{d}}=1$, while the EMA model clearly succeeds to approach gradually the pure PEO permeability. The Lewis-Nielsen (with $\mathrm{A}=2$ ) and Pal (with $\mathrm{d}=3$ ) models have been taken with $\bar{\phi}_{d}=0.64$ as should correspond to the maximum packing of spherical inclusions. It seems clear that none of them adequately fits the experimental results probably because their application domain restrict to low content of the filler within a continuous matrix. 


\section{Figure 7}

The values of N, 2.55 and 2.83, are somehow out of the range that could be expected for electrical or thermal conductivity and for permeability as far as it is well known that $\mathrm{N}$ $=2$ in a three-dimensional percolation (with an aspect ratio, $\delta=1$ ) [63], with $\delta=\mathrm{L} / \mathrm{D}, \mathrm{L}$ and $\mathrm{D}$ being the length and the diameter of the inclusions. 
In Figure 8 the EMA model is applied to the $\mathrm{CO}_{2}$ permeability of the copolyimides: BPDA-ODA-PEO(2000), BKDA-ODA-PEO(2000) and PMDA-ODA-PEO(2000). The figure shows that a change in the dianhydride has little effect in the dependence of permeability and the point of maximal increase in the effective permeability is quite similar and very close to a volume fraction of PEO $\hat{\phi}_{d} \approx 1 / 3$. This could be predicted from Equations (23) and (24) because $\lambda$ goes from $2.8 \cdot 10^{-3}$ to $17.4 \cdot 10^{-3}$ which corresponds to values of $\hat{\phi}_{d}$ going from $32.7 \%$ to $33.2 \%$.

\section{Figure 8}

Figure 9 and 10 correspond to a three phase system BKDA-ODA-PEO(6000) constituted by the pure BKDA-ODA, the amorphous $\mathrm{PEO}(6000)$ and the crystalline one. In Figure 9 the white symbols correspond to the total volume proportion of PEO(6000). The EMA model fits the results only when the actual amorphous volume proportion of $\mathrm{PEO}(6000)$ is taken into account after subtraction of the crystalline volume as obtained by DSC, [16], and the system is taken as a bi-phase system.

In Figure 10 the three phase system has been fitted to Equation (10), for three phases:

$$
\left.\begin{array}{c}
\sum_{i=1}^{3} \phi_{i} \frac{P_{i}-P_{e f f}}{P_{i}+2 P_{e f f}}=0 \\
\sum_{i=1}^{3} \phi_{i}=1
\end{array}\right\}
$$

assuming that the permeability of the crystalline PEO is zero. It is clear that as shown in Figure 10, the EMA model can predict also the permeability across three phase composite segregated copolyimides. The graph corresponds to the effective permeability for the mentioned ternary system. In the Figure, the composition of the ternary system is given in terms of the volume fractions of crystalline and amorphous PEO. The datum for the semicrystalline PEO with $29 \%$ crystallinity has been taken from Lin and Freeman [64] (see Figure 2 of this reference).

\section{Figure 9}




\section{Conclusions}

A series of aliphatic aromatic copoly(etherimide)s has been synthesized. Cast films of these copolymers have been thermally treated to ensure the phase segregation of the linear PEO chains from the aromatic moiety of the copoly(etherimide)s. The permeability of $\mathrm{O}_{2}, \mathrm{~N}_{2}, \mathrm{CO}_{2}$ and $\mathrm{CH}_{4}$ of the resulting membranes have been compared with the predictions of the main usable models adapted to the gas permeability.

The model based on the Effective Medium Approximation (EMA) succeeded to predict the main features of the experimental results. Specifically, it was able to calculate the volume fraction for the maximum increase of permeability, a common feature for all the studied segregated copolymer membranes. The model was even able to predict the permeabilities of a three phase system consisting in the aromatic (BKDA-ODA) rich phase plus the amorphous $\mathrm{PEO}(6000)$ and the crystalline $\mathrm{PEO}(6000)$ phases.

As discussed, the EMA calculations are especially applicable to composites and polycrystals in which the various components are randomly and symmetrically distributed, so that none of the components is identifiable as a host in which the others are preferentially embedded. The size and orientation of each phase are continuously changing with their proportion; this is the case with the segregated copoly(ether)imides studied here. On the other hand, there is not a constant size network to be filled and the permeabilities of the phases involved are relatively close to each other as to make percolation impossible. Nevertheless they are different enough to have a certain percolation-like sudden step in the effective permeability which is well integrated in the EMA model.

The model has the advantage of being able to be adapted for three phase (or multiphase) composites where the structure of each phase in the mixture is effectively random in nature. This fact has the added advantage of making unnecessary any consideration on the detailed phase-to-phase morphology. 


\section{Aknowledgements}

The authors thank the Ministerio de Educación y Ciencia (Plan Nacional de I+D+i) through projects MAT2011-25513 and CTQ2012-31076, Junta de Castilla y León (project VA-324A11-2) and also Acciona Agua for partial funding of this research. We are indebted also to Ministerio de Economía y Competitividad through project MAT2010-20668. A. Tena thanks CSIC for a predoctoral JAE fellowship.

\section{References}

[1] R.W. Baker, Future directions of membrane gas separation technology, Industrial \& Engineering Chemistry Research, 41 (2002) 1393-1411.

[2] R. Bounaceur, N. Lape, D. Roizard, C. Vallieres, E. Favre, Membrane processes for post-combustion carbon dioxide capture: A parametric study, Energy, 31 (2006) 25562570.

[3] E. Favre, Carbon dioxide recovery from post-combustion processes: Can gas permeation membranes compete with absorption?, Journal of Membrane Science, 294 (2007) 50-59.

[4] I. Bessonov, W.W. Wright, Polyimides: thermally stable polymers, Consultants Bureau, 1987.

[5] M.K. Ghosh, K.L. Mittal, Polyimides: Fundamentals and Applications, Marcel Dekker Incorporated, 1996.

[6] K.L. Mittal, Polyimides and Other High-temperature Polymers: Synthesis, Characterization, and Applications. Volume 5, VSP, 2009.

[7] K. Tanaka, H. Kita, M. Okano, K.-i. Okamoto, Permeability and permselectivity of gases in fluorinated and non-fluorinated polyimides, Polymer, 33 (1992) 585-592.

[8] D. Ayala, A. Lozano, J. De Abajo, C. Garcia-Perez, J. De la Campa, K.-V. Peinemann, B. Freeman, R. Prabhakar, Gas separation properties of aromatic polyimides, Journal of Membrane Science, 215 (2003) 61-73.

[9] K. Tanaka, H. Kita, K. Okamoto, A. Nakamura, Y. Kusuki, Gas permeability and permselectivity in polyimides based on 3, 3', 4, 4'-biphenyltetracarboxylic dianhydride, Journal of Membrane Science, 47 (1989) 203-215.

[10] T. Barbari, W. Koros, D. Paul, Gas transport in polymers based on bisphenol-A, Journal of Polymer Science Part B: Polymer Physics, 26 (1988) 709-727. 
[11] Y. Li, M. Ding, J. Xu, Gas separation properties of aromatic polyetherimides from its esters, Journal of applied polymer science, 63 (1997) 1-7.

[12] A. Tena, A. Marcos-Fernández, A.E. Lozano, J.G. de la Campa, J. de Abajo, L. Palacio, P. Prádanos, A. Hernández, Thermally treated copoly (ether-imide) s made from bpda and alifatic plus aromatic diamines. Gas separation properties with different aromatic diamimes, Journal of Membrane Science, 387 (2012) 54-65.

[13] A. Marcos-Fernández, A. Tena, A.E. Lozano, J.G. de la Campa, J. de Abajo, L. Palacio, P. Prádanos, A. Hernández, Physical properties of films made of copoly (etherimide) s with long poly (ethylene oxide) segments, European Polymer Journal, 46 (2010) 2352-2364.

[14] A. Gil, A. Tena, A. Marcos-Fernández, M.R. De La Viuda, A.E. Lozano, P. Prádanos, Block copolymers for gas separation: Looking for the ideal structure, in: Elsevier (Ed.) Third International Symposium: Frontiers in Polymer Science, Sitges (Spain), 2013.

[15] A. Tena, A. Marcos-Fernández, L. Palacio, P. Cuadrado, P. Prádanos, J. de Abajo, A.E. Lozano, A. Hernández, Phase Segregation and Gas Separation Properties of Thermally Treated Copoly (ether-imide) from an Aromatic Dianhydride, an Aromatic Diamine, and Various Aliphatic Diamines, Industrial \& Engineering Chemistry Research, 51 (2012) 3766-3775.

[16] A. Tena, A. Lozano, L. Palacio, A. Marcos-Fernández, P. Prádanos, J. de Abajo, A. Hernández, Gas separation properties of systems with different amounts of long poly (ethylene oxide) segments for mixtures including carbon dioxide, International Journal of Greenhouse Gas Control, 12 (2013) 146-154.

[17] A. Tena, A. Marcos-Fernandez, A.E. Lozano, J.G. De La Campa, J. De Abajo, L. Palacio, P. Pradanos, A. Hernandez, Thermally Segregated Copolymers with PPO Blocks for Nitrogen Removal from Natural Gas, Industrial \& Engineering Chemistry Research, (2013).

[18] Song Lin Liu, Lu Shao, Mei Ling Chua, Cher Hon Lau, Huan Wang, Shuai Quan, "Recent progress in the design of advanced PEO-containing membranes for CO2 removal" Progress in Polymer Science, 38(7) (2013) 1089-1120.

[19] A. Tena, A. Marcos-Fernández, L. Palacio, P. Prádanos, A.E. Lozano, J.d. Abajo, A. Hernández, On the influence of the proportion of PEO in thermally controlled phase segregation of copoly(ether-imide)s for gas separation, Journal of Membrane Science, (2013).

[20] H. Chen, Y. Xiao, T.-S. Chung, Synthesis and characterization of poly (ethylene oxide) containing copolyimides for hydrogen purification, Polymer, 51 (2010) 40774086. 
[21] Metz SJ, Mulder MHV, Wessling M. Gas-permeation properties of poly(ethyleneoxide) poly(butylene terephthalate) block copolymers, Macromolecules 237 (2004)4590-4597.

[22] A. Dufresne, J.-Y. Cavaille, Clustering and percolation effects in microcrystalline starch-reinforced thermoplastic, Journal of Polymer Science Part B Polymer Physics, 36 (1998) 2211-2224.

[23] O. Levy, D. Stroud, Maxwell Garnett theory for mixtures of anisotropic inclusions: Application to conducting polymers, Physical Review B, 56 (1997) 8035.

[24] M. Zimmer, X. Fan, J. Bao, R. Liang, B. Wang, C. Zhang, J. Brooks, ThroughThickness Thermal Conductivity Prediction Study on Nanocomposites and Multiscale Composites, Materials Sciences and Applications, 3 (2012) 131-138.

[25] B. Shimekit, H. Mukhtar, Gas permeation models in mixed matrix membranes, in: National Postgraduate Conference (NPC), 2011, IEEE, 2011, pp. 1-5.

[26] J.M. Garnett, Colours in metal glasses, in metallic films, and in metallic solutions. II, Philosophical Transactions of the Royal Society of London. Series A, Containing Papers of a Mathematical or Physical Character, (1906) 237-288.

[27] A. Eucken, Allgemeine gesetzmabigkeiten fur das warmeleitvermogen verschiedener stoffarten and aggregatzustande, Forschung Gabiete Ingenieur, 11 (1940) 6-20.

[28] D.J. Bergman, D. Stroud, Physical properties of macroscopically inhomogeneous media, Solid State Physics, 46 (1992) 147-269.

[29] J.C. Garland, D.B. Tanner, O.S. University, U.S.E. Research, D. Administration, N.S. Foundation, Electrical transport and optical properties of inhomogeneous media, Ohio State University, 1977, American Institute of Physics, 1978.

[30] D.A.G. Bruggeman, Calculation of various physical constants in heterogeneous substances. I. Dielectric constants and conductivity of composites from isotropic substances, Ann. Phys. (Paris), 24 (1935) 636.

[31] M. Haddadi, B. Agoudjil, A. Boudenne, B. Garnier, Analytical and Numerical Investigation on Effective Thermal Conductivity of Polymer Composites Filled with Conductive Hollow Particles, International Journal of Thermophysics, 34 (2013) 101112.

[32] C.J.F. Böttcher, Theory of electric polarisation, Elsevier Pub. Co., Amsterdam, the Netherlands, 1952.

[33] G. De Loor, Dielectric properties of heterogeneous mixtures, University of Leiden, Leiden, Uitgeverij Excelsior, 1956. 
[34] H. Ebadi-Dehaghani, M. Nazempour, Thermal Conductivity of Nanoparticles

Nanoparticles Technology", InTech, 2012.

[35] T. Lewis, L. Nielsen, Dynamic mechanical properties of particulate-filled composites, Journal of applied polymer science, 14 (1970) 1449-1471.

[36] L.E. Nielsen, Thermal conductivity of particulate filled polymers, Journal of applied polymer science, 17 (1973) 3819-3820.

[37] L.E. Nielsen, The thermal and electrical conductivity of two-phase systems, Industrial \& Engineering Chemistry Fundamentals, 13 (1974) 17-20.

[38] D. Kumlutaş, I.H. Tavman, M. Turhan Çoban, Thermal conductivity of particle filled polyethylene composite materials, Composites Science and Technology, 63 (2003) 113-117.

[39] B. Weidenfeller, M. Höfer, F.R. Schilling, Thermal conductivity, thermal diffusivity, and specific heat capacity of particle filled polypropylene, Composites Part A: applied science and manufacturing, 35 (2004) 423-429.

[40] D. Kumlutaş, I.H. Tavman, A numerical and experimental study on thermal conductivity of particle filled polymer composites, Journal of Thermoplastic Composite Materials, 19 (2006) 441-455.

[41] R. Pal, On the Lewis-Nielsen model for thermal/electrical conductivity of composites, Composites Part A: applied science and manufacturing, 39 (2008) 718-726.

[42] H. Davis, L. Valencourt, C. Johnson, Transport processes in composite media, Journal of the American Ceramic Society, 58 (1975) 446-452.

[43] R. Landauer, The electrical resistance of binary metallic mixtures, Journal of Applied Physics, 23 (1952) 779-784.

[44] P. Karayacoubian, Effective thermal conductivity of composite fluidic thermal interface materials, in: Applied Science In Mechanical Engineering, Univ. Waterloo, Ontario, Canada 2006.

[45] X. Colin Tong Advanced Materials for Thermal Management of Electronic Packaging, Sprienger series in advanced microelectronics 30, Springer Science+Business Media, New York, USA, 2011.

[46] D.L. Johnson, P.N. Sen, Physics and chemistry of porous media, American Institute of Physics, 1984.

[47] F. Levy, A modified Maxwell-Eucken equation for calculating the thermal conductivity of two-component solutions or mixtures, International Journal of Refrigeration, 4 (1981) 223-225. 
[48] J. Wang, J.K. Carson, M.F. North, D.J. Cleland, A new approach to modelling the and Mass Transfer, 49 (2006) 3075-3083.

[49] T.-S. Chung, L.Y. Jiang, Y. Li, S. Kulprathipanja, Mixed matrix membranes (MMMs) comprising organic polymers with dispersed inorganic fillers for gas separation, Progress in Polymer Science, 32 (2007) 483-507.

[50] R. Mahajan, W.J. Koros, Mixed matrix membrane materials with glassy polymers. Part 1, Polymer Engineering \& Science, 42 (2002) 1420-1431.

[51] T.T. Moore, R. Mahajan, D.Q. Vu, W.J. Koros, Hybrid membrane materials comprising organic polymers with rigid dispersed phases, AIChE journal, 50 (2004) 311-321.

[52] J. Felske, Effective thermal conductivity of composite spheres in a continuous medium with contact resistance, International Journal of Heat and Mass Transfer, 47 (2004) 3453-3461.

[53] B. Shimekit, H. Mukhtar, T. Murugesan, Prediction of the relative permeability of gases in mixed matrix membranes, Journal of Membrane Science, 373 (2011) 152-159.

[54] S.R. Broadbent, J.M. Hammersley, Percolation processes I. Crystals and mazes, in: Proc. Cambridge Philos. Soc, 1957, pp. 629-641.

[55] G. Grimmet, Percolation, vol. 321 in A Series of Comprehensive Studies in Mathematics, Springer, Berlin, Germany, 1999.

[56] D. Bigg, The effect of compounding on the conductive properties of EMI shielding compounds, Advances in Polymer Technology, 4 (1984) 255-266.

[57] J. Clerc, G. Giraud, J. Laugier, J. Luck, The electrical conductivity of binary disordered systems, percolation clusters, fractals and related models, Advances in Physics, 39 (1990) 191-309.

[58] Y.P. Mamunya, V. Davydenko, P. Pissis, E. Lebedev, Electrical and thermal conductivity of polymers filled with metal powders, European Polymer Journal, 38 (2002) 1887-1897.

[59] W. Tian, R. Yang, Phonon transport and thermal conductivity percolation in random nanoparticle composites, Computer modeling in engineering and sciences, 24 (2008) 123.

[60] I.U.K. Godovskiĭ, V.P. Privalko, Thermal and electrical conductivity of polymer materials, Springer, New York, USA, 1995.

[61] G. Zhang, Y. Xia, H. Wang, Y. Tao, G. Tao, S. Tu, H. Wu, A percolation model of thermal conductivity for filled polymer composites, Journal of composite materials, 44 (2010) 963-970. 
[62] M. Kole, D. Tripathi, T.K. Dey, Percolation based enhancement in effective thermal conductivity of HDPE/LBSMO composites, Bulletin of Materials Science, 35 (2012) 601-609.

[63] D. Stauffer, A. Aharony, Introduction to Percolation Theory, Taylor \& Francis Group, London. UK, 1994.

[64] H. Lin, B.D. Freeman, Gas solubility, diffusivity and permeability in poly (ethylene oxide), Journal of Membrane Science, 239 (2004) 105-117. 
Figure 1.- Structure of the aromatic and aliphatic components of the copolyimide thermally segregated membranes studied here along with their densities. ( $\left.{ }^{a}\right)$ Data from the manufacturer and $\left({ }^{b}\right)$ Tanaka et al. [7]. Volume fractions $\phi_{d}$ can be evaluated from weight fractions $\omega_{d}$ by: $\phi_{d}=\left(\omega_{d} / \rho_{d}\right) /\left[\left(\omega_{d} / \rho_{d}\right)+\left(1-\omega_{d}\right) / \rho_{c}\right]$. $\rho_{d}$ is the density of filler and $\rho_{c}$ is the density of the matrix.

Figure 2.- Comparison of some models for the effective permeability of $\mathrm{CO}_{2}$ for a maximum thermally segregated polyimide. The Bruggeman model corresponds to Equation (2) with $d=3$. The EMA model corresponds to the s-shaped continuous thick line.

Figure 3.- Plot of Equation (1724) showing that there is a range from $\phi_{d}=1 / 3$ to $2 / 3$ that can't correspond to the inflexion point in $\mathrm{P}_{\text {eff. }}$ The inflexion point for the Levy model should be placed in $\phi_{\mathrm{d}}=1 / 2$.

Figure 4.- Effective permeability of: $\mathrm{O}_{2}, \mathrm{~N}_{2}$ and $\mathrm{CH}_{4}$ for a thermally segregated polyimide. The lines correspond to the EMA model. Vertical lines correspond to the maximum increase in permeability.

Figure 5.- Effective permeability of $\mathrm{O}_{2}$ and predictions of some models for low content of a dispersed phase. The Lewis-Nielsen $(A=2)$ and the Pal $(d=3)$ models have been shown for $\bar{\phi}_{d}=0.64$. The EMA model predictions are shown for the sake of comparison. Vertical lines correspond to the maximum increase in permeability

Figure 6.- Effective permeability of $\mathrm{N}_{2}$ and predictions of the Pal model (with $\mathrm{d}=3$ ) for three different $\bar{\phi}_{d}$. The double vertical lines correspond to the maximum increase in permeability while the other vertical lines correspond to the asymptotes of the Pal model. 
Figure 7.- Effective permeability of $\mathrm{CH}_{4}$ and predictions of the percolation model of Zhang. The white cross corresponds to a fixed pair $\phi_{t}$ and $P_{t}$ while the gray one corresponds to values obtained for $\phi_{\mathrm{t}}$ and $\mathrm{P}_{\mathrm{t}}$ after fitting.

Figure 8.- Effective permeability of $\mathrm{CO}_{2}$ for thermally segregated polyimides with different dianhydrides. The lines correspond to the EMA model.

Figure 9.- Effective permeability of $\mathrm{CO}_{2}$ for a partially crystalline PEO within a BKDA-ODA matrix at $30^{\circ} \mathrm{C}(\mathrm{O})$. When the fractions of real amorphous PEO $(\oplus)$ are taken into account EMA predictions reproduce the experimental results. The line correspond to the EMA model. At $50^{\circ} \mathrm{C}(\mathbf{)})$ there is no crystallinity and the EMA predictions are again quite good.

Figure 10.- Effective permeability of $\mathrm{CO}_{2}$ for a three-phase system of crystalline and amorphous PEO in a BKDA-ODA matrix and the predictions of EMA. 


\title{
Prediction of Gas Permeability of Block-Segregated Polymeric Membranes by an Effective Medium Model.
}

\author{
AlbertoTena $^{1,3}$, Mónica de la Viuda ${ }^{1}$, Laura Palacio ${ }^{1,2}$, Pedro Prádanos ${ }^{1,2}$, Ángel \\ Marcos-Fernández ${ }^{1,3}$, Ángel E. Lozano ${ }^{1,3}$ and Antonio Hernández ${ }^{1,2}$ \\ ${ }^{1}$ Smap UA-UVA-CSIC, Parque Científico Universidad de Valladolid, Paseo de Belén, s/n, 47011 \\ Valladolid, Spain. \\ ${ }^{2}$ Dept. Física Aplicada, Facultad de Ciencias, Universidad de Valladolid, Paseo de Belén, 7, 47011 \\ Valladolid, Spain \\ ${ }^{3}$ Instituto de Ciencia y Tecnología de Polímeros, CSIC, Juan de la Cierva 3, 28006 Madrid, Spain.
}

\section{Summary}

A complete series of aliphatic aromatic copoly(ether-imide)s, based on aromatic dianhydrides (BPDA, $B K D A$ or PMDA) and mixtures of an aromatic diamine (ODA) and an aliphatic diamine terminated poly(ethylene oxide) PEO(2000) (Mw=2000 $\mathrm{g} / \mathrm{mol})$ or PEO(6000) (Mw=6000 $\mathrm{g} / \mathrm{mol})$, has been synthesized using different PEO contents. Cast films of these copolymers have been thermally treated to ensure the segregation of the linear PEO chains from the aromatic portion of these copoly(ether-imide)s.

Gas permeability $\left(\mathrm{O}_{2}, \mathrm{~N}_{2}, \mathrm{CO}_{2}\right.$ and $\left.\mathrm{CH}_{4}\right)$ of membranes, made from these copolymers with different proportions of $\mathrm{PEO}$, were compared with the predictions of various electrical or thermal conductivity models adapted to gas permeability. Several of these models, from Maxwell-Garnett to percolation theory, proved to be inaccurate. Nevertheless, the model based on the Effective Medium Approximation (EMA) is proved here to succeed in predicting the main features of the experimental results for all mixture proportions, taking as the starting point the sole input of pure homopolymer permeabilities. Specifically, this later method was able to calculate the volume fraction for the maximum increase of permeability, a common feature for all the studied segregated copolymer membranes. The model was even able to predict the permeabilities of a three phase system consisting in the aromatic (BKDA-ODA) phase plus a mixture of amorphous PEO(6000) and crystalline PEO(6000).

Keywords: Copoly(ether-imide) membrane; Phase segregation; Thermal treatment; Gas separation; Effective Medium Approximation, Percolation, Maxwell-Garnett Models.

\section{*membrana@termo.uva.es}

Tel.: $\quad 34-983423134$

Fax.: 34-983423013 


\section{Introduction}

Nowadays, the role of polymeric membranes applied to gas separation is being more and more important. Although some of these materials have been used in industrial separations[1], it is well known that a lot of research is still necessary to discover or refine materials having an equilibrium of properties making them suitable to be employed in applications demanded nowadays by the industry. In all cases, an adequate balance of high permeability and good selectivity must be achieved [2-3].

In order to be useful in gas separation applications where one or several of the gases to separate-be separated is a condensable gas such as $\mathrm{CO}_{2}$ or $\mathrm{CH}_{4}$, the polymeric membrane should show a preferential affinity for them. In addition, these membranes must have good mechanical and thermal resistance.

Glassy polymers and in particular polyimides are well known by their excellent thermal oxidative stability, good organic solvent resistance and exceptional mechanical properties, along with an extraordinary ability to separate complex mixtures of gases in diverse applications [4-6]. Thus, it has been widely recognized that the use of aromatic polyimides is one of the best alternatives to be chosen among all the polymeric membranes for gas separation applications [7].

Typically, these materials have good selectivity and not very high permeability [8-9]. It could therefore be interesting to increase the affinity of the material matrix for condensable gases such as $\mathrm{CO}_{2}$, or $\mathrm{CH}_{4}$ as one of the most effective approaches.

Aromatic-aliphatic block-copolymers usually combine a hard block and a soft block. The hard block can be formed by a polymer with well-packed and highly rigid structures; as a result it forms a glassy phase with usually low free volume. In contrast, the soft block can consist in a polymer with more flexible, low $\mathrm{T}_{\mathrm{g}}$, chains, which can form a rubbery phase having high free volume. Also, when aromatic-aliphatic block copolymers are phase-separated, for instance, by an adequate thermal treatment, their glassy polymer parts could provide mechanical support. The rubbery segments, due to the nature of the flexible chain structure, should allow an efficient transport of gas, 
giving a good permeability to the material [10-11]. Among all the block copolymers,

The synthesis of new PEO systems using different aromatic diamines showed that an increase of rigidity in the hard part promoted the phase segregation process [12]. When the temperature of treatment of the films increased, phase segregation improved [13]. Analogous trends have been proved when diverse types of aromatic dianhydrides of different rigidity were employed [14]. These processes depended also on the type of polyether used, because the phase segregation and the final properties of the membranes can also vary depending on the nature of the soft part [15].

Mechanical properties of the membranes tended to worsen when the amount of polyether increased, because the amount of the hard part (which provides the mechanical strength of the final polymer) decreased. At the same time, the segregation and the permeation properties improved $[16-17,18]$.

Polyethers such as PEO can experience easy crystallization processes when the molecular weight exceeds a certain value [16]. In gas separation this increase of crystallinity is undesirable because ordered structures have low or null permeation capacity. When PEO is in amorphous state, it can be said that the permeability increases with the length of the aliphatic part, mainly due to the improvement in phase segregation [16]. It is clearly important to find a good balance between the hard and the soft block segments in order to provide good permeability without having a dramatic loss in mechanical properties.

Many composite systems, as for example mixed matrix membranes, MMM, have been modeled to correlate the overall permeability with those of the component phases by applying Maxwell-Garnett, MG, based treatments. Herein, we have applied this treatment to phase segregated membranes and we have found difficulties to model, within this theoretical frame, the range of intermediate concentrations. In this paper it is demonstrated that more symmetric schemes as the effective medium approximations, EMA, reproduce well enough the composition ranges where none of the phases, forming the material, can be considered as dispersed in a continuous matrix. 


\section{Experimental}

The segregated copoly(etherimide)s whose permeabilities are modeled here have been synthetized, thermally segregated and characterized previously by our group [12, 15-16, 19]. A brief summary of these aspects is provided below.

\subsection{Chemicals}

3,3',4,4'- Biphenyltetracarboxylic dianhydride (BPDA); 3,3',4,4'-benzophenone tetracarboxylic dianhydride (BTDA or BKDA); pyromellite dianhydride (PMDA), and 4,4'-oxydianiline (ODA) were purchased from Aldrich. These products were purified by sublimation at high vacuum just before use. Polyoxyethylene bis(amine) (Jeffamine ED2003, $n=41$ ) with nominal molecular weight of $2000 \mathrm{~g} / \mathrm{mol}$, was kindly donated given by Huntsman ${ }^{\circledR}$ (Holland) (PEO-2000 from here on). $\alpha, \omega$-Diamine-poly(ethylene oxide) with nominal molecular weight of $6000 \mathrm{~g} / \mathrm{mol}$, were obtained from Kawaken Fine Chemicals Co., Ltd. (Tokyo, Japan), (PEO-6000 from here on). These polyethers were dried at $70{ }^{\circ} \mathrm{C}$ in vacuum for 5 hours and stored in a desiccator at vacuum until use. Anhydrous N-methylpyrrolidinone (NMP), used as the polymerization solvent, was purchased from Sigma-Aldrich Co.

\subsection{Synthesis of copoly(ether-imide)s}

The samples were synthesized by combination of a dianhydride (BPDA, BKDA or PMDA) with mixtures of an aromatic diamine (ODA), and diverse proportions of an aliphatic diamine (PEO). The corresponding copoly(ether-imide)s will be designated by adding $\mathrm{cPI}$ to the $\mathrm{w} / \mathrm{w}$ percentage of the aliphatic proportion.

Diamine-terminated poly(oxyethylene oxide $)-\operatorname{PEO}(2000)$ or $\mathrm{PEO}(6000)-(x \mathrm{mmol})$, and 4,4'-oxydianiline (ODA) ( $y \mathrm{mmol}$ ) in several weight ratios were dissolved in anhydrous NMP $(5 \mathrm{mmol}(x+y) / 10 \mathrm{~mL})$ in a $100 \mathrm{~mL}$ three-necked flask blanketed with nitrogen. 
Then, the reaction mixture was cooled down to $0{ }^{\circ} \mathrm{C}$, and under mechanical stirring, a

In Figure 1 the corresponding structures of the aromatic and aliphatic homopolymers corresponding to the copoly(ether-imide)s synthetized are shown along with their densities

\section{Figure 1}

\subsection{Preparation of the copolyimide dense films.}

The resultant viscous copolyamic acid solution was diluted with NMP to the appropriate viscosity for casting, filtered through a nominal \#1 fritted glass funnel, degassed, and cast onto a leveled glass plate. The resulting film was covered with a conical funnel to avoid fast evaporation of the solvent, dried at $80{ }^{\circ} \mathrm{C}$ overnight, and finally treated at several temperatures from 160 to $250^{\circ} \mathrm{C}\left(275^{\circ} \mathrm{C}\right.$ for the BKDA containing films) for 6 hours until $200^{\circ} \mathrm{C}$ and 30 min over this temperature in a vacuum oven, in order to achieve a complete imidization. Films of copolymers having 50-70 $\mu \mathrm{m}$ in thickness were obtained. After that, thermal treatments under an inert atmosphere were carried out at different temperatures.

\subsection{Characterization of the membranes.}

All the copoly(eherimide)s studied here were treated at different temperatures (as mentioned above) and the resulting films extensively characterized [12-19]. They were and-Phase segregation was confirmed by Small-Angle X-ray Scattering (SAXS) observing that this process increases with temperature. Glass transition temperatures, crystallinity and mechanical properties were obtained by using Differential Scanning Calorimetry (DSC), Thermo Gravimetric Analysis (TGA), Thermo Mechanical Analysis (TMA) and Dynamic Mechanical Analysis (DMA). All the films showed good mechanical properties. 
Permselectivities were obtained by using "time lag" permeators at 3 bar and $30{ }^{\circ} \mathrm{C}$ (some measurements were done at $50{ }^{\circ} \mathrm{C}$ when crystallinity was present in the case of PEO-6000).

\section{Theory}

The membranes composed by two or more different phases should have permeability values placed between those for pure phases. Copolyimides consisting in an aromatic polyimide part (hard segments) and an aliphatic polyether one (soft PEO chains) should be modeled as a system formed by two intermixed phases.

It has been generally $[16,20,21]$ found that for intermediate volume fractions there is a range where none of the Maxwell-Garnett predictions are accurate. It has been also noticed that low PEO proportions are well predicted by the Maxwell-Garnett model assuming that PEO is the dispersed phase although for high PEO proportions the Maxwell-Garnett model with PEO as the continuous phase fits better the experimental results [20]. Of course there is a range where neither the PEO rich nor the aromatic rich phases can be taken as disperse or (discontinuous). This intermediate range has resisted all attempts to be predictable or even understood [19]. Among the attempts made we can mention the addition of an ad hoc parameter, so called "chain immobilization factor", that should be evaluated as a function of composition to take into account the differences between the predictions of Maxwell-Garnett model for small fractions of one of the phases and the experimental results [21]. Other possibility is to patch both the Maxwell-Garnet predictions corresponding to PEO as disperse and to PEO as continuous phase by introducing a threshold value for the composition that again has to be adapted "ad hoc" to the experimental results [19].

A revision will be made here of some of the main models that can be used to predict permeabilities for different proportions of a multiphase gas separation membrane. This revision cannot be exhaustive as far as there are a huge plethora of models more or less similar to each other which were originally proposed for electric or thermal conductivity or for mechanical properties [22-24]. The models will, of course, be adapted to gas 
permeation attending to the well-known analogy existent between the transport processes [25].

\subsection{Maxwell-Garnett Models}

The Maxwell-Garnett approximation, [26-27] also known as the Clausius-Mossotti approximation, is one of the most widely used methods for calculating the bulk dielectric properties of inhomogeneous materials [28-29]. It is useful when one of the components can be considered as a host in which inclusions of the other component or components are embedded. It involves an exact calculation of the field induced in the uniform host by a single spherical or ellipsoidal inclusion and an approximate treatment of its distortion by the electrostatic interaction between the different inclusions. This distortion is caused by the charge dipoles and higher multipoles induced in the other inclusions. The induced dipole moments cause the longest range distortions and their average effect is included in the Maxwell approximation which results in a uniform field inside all the inclusions.

These approaches have been extensively used to study the properties of two-component mixtures in which both, the host and the inclusions, are isotropic materials with scalar dielectric coefficients. Nevertheless, applying relaying inthe known analogies of transport phenomena, this theoretical frame has been used to model: electrical conductivity, thermal conductivity and elasticity parameters. They have been also extensively used for permeation modeling.

For gas separation processes, it has been demonstrated that systems consisting in a disperse phase embedded in a continuous matrix can be described in terms of these models that have been called Maxwell-Garnett or Maxwell-Eucken models. Within the frame of these theoretical schemes, the effective permeability is:

$$
P_{e f f}=P_{c}\left[\frac{P_{d}+2 P_{c}-2\left(P_{c}-P_{d}\right)}{P_{d}+2 P_{c}+\left(P_{c}-P_{d}\right)}\right]
$$


Where $P_{\text {eff }}$ is the effective permeability, $P_{c}$ and $P_{d}$ are the permeabilities of the continuous and disperse phase, respectively, and $\phi_{\mathrm{d}}$ is the volume fraction of the dispersed phase in the block copolymer.

In our study, of course the continuous phase can be the aromatic or the aliphatic rich portions depending on the composition of the copolymer. For this reason, it should be necessary to make two different predictions depending on which segment is taken as the continuous phase or the disperse one. This is necessary because Equation (1) is not symmetric and if the roles of the filler and the continuous phase are swapped, different results are obtained.

Bruggeman [30] introduced a popular approximation that is now widely known as the differential scheme or the Bruggeman equation. Bruggeman proceeds from the premise that the fields of neighboring particles can be taken into account by adding the dispersed particles incrementally, taking the surrounding medium to be the existing composite at each stage.

Translated to gas permeability, the Bruggeman equation says that the effective permeability of an inhomogeneous medium is given by:

$\frac{P_{d}-P_{e f f}}{P_{d}-P_{c}}\left(\frac{P_{c}}{P_{\text {eff }}}\right)^{1 / d}=1-\phi_{d}$

where $\mathrm{d}=3$ for spherical fillers [31].

The Bruggeman equation is appropriate for composites containing spherical particles with a very wide size distribution. Ideally, the size distribution should be wide enough so that any two spheres of comparable size are far from each other. Note that Equation (2) needs to be solved numerically and it is also asymmetric, in the sense commented when referring to Equation (1), i.e. it is not single valued.

Some models have been applied without any pursued symmetry between filler and continuous matrix because they are intended for low content of filler within a continuous matrix. Most of these models lead to divergences in permeability for filler contents over a certain value. They belong to this class, for example, the models due to 
Bruggeman [30], Bötcher [32] and De Loor [33] and corresponding to Equations (3) to

(5) [34]:

$$
\begin{aligned}
& P_{e f f}=P_{c}\left(1-\phi_{d}\right)^{-3} \\
& P_{e f f}=P_{c}\left(1-\phi_{d}\right)^{-1} \\
& P_{e f f}=P_{c}\left(1-\phi_{d}\right)\left(1-2 \phi_{d}\right)^{-1}
\end{aligned}
$$

The Lewis and Nielsen model [35-37], originally proposed for the elastic modulus of particulate composites, gives:

$$
\begin{aligned}
& P_{e f f}=P_{c}\left[\frac{1+A \phi_{d} \frac{\left(P_{d} / P_{c}\right)-1}{\left(P_{d} / P_{c}\right)+A}}{1-\psi \phi_{d} \frac{\left(P_{d} / P_{c}\right)-1}{\left(P_{d} / P_{c}\right)+A}}\right] \\
& \psi=1+\phi_{d}\left(\frac{1-\bar{\phi}_{d}}{\bar{\phi}_{d}^{2}}\right)
\end{aligned}
$$

The values of A and $\bar{\phi}_{d}$ were given for many geometric shapes and orientations [38-40]. A is 2 for spheres in a unidirectional transport, $\bar{\phi}_{d}$ is 0.64 for random close packing of uniform spheres. $\bar{\phi}_{d}$ is the maximum $\phi_{d}$ that is the maximum packing volume fraction for a given inclusion size and shape.

The Pal model [41] assumes that:

$$
\frac{P_{d}-P_{e f f}}{P_{d}-P_{c}}\left(\frac{P_{c}}{P_{e f f}}\right)^{1 / d}=\left(1-\frac{\phi_{d}}{\bar{\phi}_{d}}\right)^{\bar{\phi}_{d}}
$$

where $d=3$ as in Equation (2) for spheres like for the original work of Bruggeman (Equation (2)). Pal originally proposed this expression for the thermal conductivity of particulate composites using a differential scheme and taking into account the packing difficulty of particles and modifying the Bruggeman model.

Equations (6) to (8) belong also to the divergent or low filler fraction models. 


\subsection{Effective Medium Theory}

Actually, when both phases are not continuous and there is a random dispersion of them, the Maxwell-Garnett approach is not reasonable. Within the frame of the Maxwell-Garnett's model, the continuous and discontinuous phases must be first identified because Equations (1) to (8) are not symmetric as mentioned. Systems where the formation of an internal network in the structure is observed are more accurately represented with the effective medium theory (EMT) that treats the contributions of each phase equally. As the equation of Maxwell-Garnett, EMT is derived from the solution of the Laplace equation applied to a single sphere surrounded by a continuous medium, and subjected to a steady-state concentration gradient around the spheres. Maxwell-Garnett assumed that the local distortions to the concentration distributions around the dispersed spheres did not affect their neighbors. The essence of EMT, however, lies in the assumption that for a completely random distribution of components, the effect of local distortions to the concentration distribution caused by individual inclusions could be averaged in such way that over a sufficiently large volume (or ensemble) the concentration distribution within the material could be approximated by a material having an uniform concentration distribution and permeability $\mathrm{P}_{\text {eff }}$ [42-43].

Effective medium approximations or effective medium theory (sometimes abbreviated as EMA or EMT) are physical models that describe the macroscopic properties of composite materials, which are developed from averaging the multiple values of the constituents that directly make up the composite material. At the constituent level, the values of the materials vary and are inhomogeneous. Precise calculation of the many constituent values is nearly impossible. The EMA methodologies are based [30] on a self-consistent procedure in which a grain of one of the components is assumed to have a convenient shape (usually spherical or ellipsoidal) and to be embedded in an effective medium whose properties are determined self-consistently [29, 43]. EMA treats equally both the components as shown in its fundamental equation, [44, 45]:

$\phi_{d} \frac{P_{d}-P_{e f f}}{P_{d}+2 P_{e f f}}+\phi_{c} \frac{P_{c}-P_{e f f}}{P_{c}+2 P_{e f f}}=0$ 
Note that this frame can be extended to $n$ phases by using:

$\sum_{i=1}^{n} \phi_{i} \frac{P_{i}-P_{e f f}}{P_{i}+2 P_{e f f}}=0$

Although the distinction of dispersed and continuous phases losses its meaning as far as the two phases are treated in the same way and the roles of both the phases could be interchanged and the predicted overall permeability would not change, we will continue naming disperse phase to the PEO domains and continuous phase to the aromatic rich one. Equation (9) can be solved to:

$P_{e f f}=\frac{1}{4}\left(\gamma+\sqrt{\gamma^{2}+8 P_{d} P_{c}}\right)$

with

$\gamma=\left(3 \phi_{d}-1\right) P_{d}+\left(3 \phi_{c}-1\right) P_{c}$

of course using the identity $\phi_{c}=1-\phi_{d}$. All these EMA calculations are particularly appropriate for composites and polycrystals in which the grains of the various components are randomly and symmetrically distributed, so that none of the components is identifiable as a host in which the others are preferentially embedded.

Because of the asymmetry of the assumed geometry, and the approximation, the results are likely to differ somewhat from the unevaluated (symmetric) EMA. This geometrical distinction may be easily understood by noting that the EMA and MG approximations are exact for two different microgeometries [28]. The EMA becomes exact in a hierarchical geometry where the two components play symmetrical geometric roles [46]. In contrast, the MG approach is exact for a geometry where the entire space is filled with equal spheres, each with identical ratio, such that one component is the core material and the other is the coating material (the host).

\subsection{Modifications of the Maxwell-Garnett models}

Modifications of the Maxwell-Garnett model have been done in two main directions. Firstly, the original asymmetric model has been modified to be symmetrical. And also the model has been modified to take into account the appearance of different phases 
corresponding, for example, to the interfaces between the matrix and the disperse phases or to take into account the differences of: size, isotropy etc. In this work, we will only mention in some detail the modifications addressed to make the approach symmetric and to take into account the possible presence of more phases.

The Levy model was originally proposed as a purely mathematical averaging of the Maxwell-Eucken model with each of the two phases being treated as continuous [47]. This model was recently given a physical interpretation by Wang et al. [48]. In terms of gas permeability the model states that:

$$
\begin{aligned}
& P_{e f f}=P_{c}\left[\frac{P_{d}+2 P_{c}-2\left(P_{c}-P_{d}\right) F}{P_{d}+2 P_{c}+\left(P_{c}-P_{d}\right) F}\right] \\
& F=\frac{(2 / g)-1+2 \phi_{d}-\sqrt{\left[(2 / g)-1+2 \phi_{d}\right]^{2}-\left(8 \phi_{d} / g\right)}}{2} \\
& g=\frac{\left(P_{c}-P_{d}\right)^{2}}{\left(P_{d}+P_{c}\right)^{2}+\frac{P_{d} P_{c}}{2}}
\end{aligned}
$$

which is symmetrical and single valued although, as we will see below, its symmetry could be inadequate in some cases.

In order to treat three-phase-systems the Maxwell-Garnett model has been modified as:

$$
P_{e f f}=P_{c}\left[\frac{P_{d}+2 P_{c}-2\left(P_{c}-P_{d}\right) F}{P_{d}+2 P_{c}+\left(P_{c}-P_{d}\right) F}\right]=P_{c}\left[\frac{2\left(1-\phi_{d}\right)+\left(1+2 \phi_{d}\right)\left(P_{d} / P_{c}\right)}{\left(2+\phi_{d}\right)+\left(1-\phi_{d}\right)\left(P_{d} / P_{c}\right)}\right]
$$

with

$$
P_{d}=P_{i n}\left[\frac{2\left(1-\phi_{k}\right)+\left(1+2 \phi_{k}\right)\left(P_{k} / P_{i n}\right)}{\left(2+\phi_{k}\right)+\left(1-\phi_{k}\right)\left(P_{k} / P_{i n}\right)}\right]
$$

$P_{\text {eff }}$ is the permeability of the ensemble continuous phase plus disperse phase, $P_{d}$ is the permeability of a single core plus interfacial shield particle and $\mathrm{P}_{\text {in }}$ is the permeability of the interfacial shield covering the particle core that has a permeability $\mathrm{P}_{\mathrm{k}} . \phi_{\mathrm{d}}$ is the 
volume fraction of inclusions and $\phi_{\mathrm{k}}$ is the fraction of the core in the core+shield system. This model has been developed to take into account the existence of such shields covering inclusions in mixed matrix membranes [49-51].

The Felske model [52] has been proposed for such complex (three phases) inclusions. This model gives:

$$
\begin{aligned}
& P_{e f f}=P_{c}\left[\frac{2\left(1-\phi_{d}\right)+\left(1+2 \phi_{d}\right)(\alpha / \beta)}{\left(2+\phi_{d}\right)+\left(1-\phi_{d}\right)(\alpha / \beta)}\right] \\
& \alpha=\frac{\left(2+\delta^{3}\right) P_{k}-2\left(1-\delta^{3}\right) P_{i n}}{P_{c}} \\
& \beta=1+2 \delta^{3}-\left(1-\delta^{3}\right) \frac{P_{k}}{P_{i n}}
\end{aligned}
$$

And $\delta$ is the ratio of outer-shield to core radii.

The Felske model gives almost the same results than the modified Maxwell-Garnett model and although it is somewhat simpler, it has the same limitations than the original Maxwell-Garnett model [53].

As done by Lewis and Nielsen, $\bar{\phi}_{d}$ can be introduced in the Felske model to give [36, 53]:

$$
P_{e f f}=P_{c}\left[\frac{1+2 \frac{\alpha-\beta}{\alpha+2 \beta} \phi_{d}}{1+\frac{\alpha-\beta}{\alpha+2 \beta} \psi \phi_{d}}\right]
$$

with $\psi$ given by Equation (7).

\subsection{Percolation Models}

The original problem was proposed [54] for the fluid flow in a porous medium. It has been applied to many other related problems including many phenomena in physics, chemistry and materials science [55]. 
A two phase system formed by inclusions dispersed in a continuous matrix can be modeled by a (3D) lattice in which each site can be occupied or empty with certain complimentary probabilities. In mathematics, percolation theory describes the behavior of connected clusters in such a random graph. In terms of gas permeability, an occupied site has a permeability $P_{d}$ while an unoccupied site has a permeability $P_{c}$. The fundamental premise of percolation theory is the appearance of a sharp increase in the effective permeability of the disordered media at a critical volume fraction known as the percolation threshold $\phi_{\mathrm{t}}$ at which long-range connectivity of the system appears.

When $P_{d} \neq 0, \mathrm{P}_{\mathrm{c}}=0$, and $\phi_{\mathrm{d}}<\phi_{\mathrm{t}}$, no macroscopic conducting pathway exists and no permeation happens through the composite phase. When $\phi_{\mathrm{d}}>\phi_{\mathrm{t}}$, however, the system becomes permeable as a connected path appear through the composite material. The existence of a critical percolation threshold for electrical conductivity has been demonstrated for a wide variety of fillers, all at concentrations below the maximum packing fraction, $\bar{\phi}_{d}$ [56]. The percolation threshold in an actual composite material is in general a function of the lattice structure of the phases, and ranges from $\phi_{t} \approx 0.2$ for a face-centered cubic arrangement to $\phi_{t} \approx 0.7$ for a honeycomb arrangement and it can be exactly calculated for certain simple lattices $[44,45]$.

Electrical conductivity has been treated frequently within percolation models [57] but also thermal conductivity has been modeled as percolative [58-59]. However, many researchers have reported that no percolation appears in thermal conductivity [60]. It may be noted that in contrast to the electrical conductivity, the concentration dependence of thermal conductivity does not show any swift leap that could be interpreted as a percolation threshold. This is because thermal conductivity of the dispersed filler is usually comparable to that of the polymer matrix. Given that this is even more certain for the gas permeabilities through disperse and continuous phases in a composite, we could find difficult to justify the appearance of percolation in the systems to be studied here. Nevertheless, as will be seen below, there is actually a sudden increase in the effective permeability of our two phase systems, thus we will adapt and try to use the most promising percolation model proposed for the thermal conductivity. 
This model was proposed by Zhang et al. [61-62] and translated to permeability says that:

$P_{e f f}=P_{d}\left(\frac{P_{t}}{P_{d}}\right)^{\left[\frac{1-\phi_{d}}{1-\phi_{t}}\right]^{N}}$

$\mathrm{P}_{\mathrm{t}}$ is the permeability at the threshold (percolation) composition given by $\phi_{\mathrm{t}}$ and $\mathrm{N}$ is the percolation exponent. This model contains actually 3 parameters to be fitted. Although the values of $\mathrm{P}_{\mathrm{t}}$ and $\phi_{\mathrm{t}}$ can be obtained by an analysis of the experimental data, $\mathrm{n}$ has to be fitted or evaluated for some fixed point.

\section{Results and Discussion}

In Figure 2 the $\mathrm{CO}_{2}$ permeability of the copolyimide BPDA-ODA-PEO(2000) thermally treated to $250{ }^{\circ} \mathrm{C}$ as a function of the volume fraction of PEO $\left(\phi_{d}\right)$ is shown. The predictions of Equations: (1) (Maxwell-Garnett); (2) (Brugeman); (9), (11) and (12) (EMA) and of Equations (13), (14) and (15) (Levy) are shown. It seems clear that the EMA model reproduces well all the main features of experimental data. It seems clear that the presence of an inflexion point in the experimental data is only predicted by the EMA and the Levy models. The vertical double line in Figure 2 corresponds to the volume fraction of PEO for this inflexion point.

\section{Figure 2}

When Equations (11) and (12) are taken into account it is seen that an inflexion point always appears, within the EMA model when $\log \mathrm{P}_{\mathrm{eff}}$ is plotted versus $\phi_{\mathrm{d}}$. This inflexion point can be calculated by solving $\mathrm{d}^{2} \log \left(\mathrm{P}_{\mathrm{eff}}\right) / \mathrm{d}_{\mathrm{d}}{ }^{2}=0$ and corresponds to the volume fraction of the disperse phase at which $\mathrm{P}_{\text {eff }}$ increases at its maximum pace. This happens when:

$\hat{\phi}_{d}=\frac{1-2 \lambda}{3(1-\lambda)}$ or for all $\phi_{\mathrm{d}}$ when $\lambda=1$

Here

$\lambda=\frac{P_{d}}{P_{c}}$

59 
It is worth remembering that the roles of continuous and disperse phases could be interchanged.

The behavior of the function given in Equation (23) is shown in Figure 3. Note that the maximum increase of $\mathrm{P}_{\mathrm{eff}}$ happens for $\hat{\phi}_{d} \approx 1 / 3$ if $\mathrm{P}_{\mathrm{d}}>>\mathrm{P}_{\mathrm{c}}\left(\right.$ or $\hat{\phi}_{d} \approx 2 / 3$ if $\mathrm{P}_{\mathrm{c}}>>\mathrm{P}_{\mathrm{d}}$ ). Moreover only when $\mathrm{P}_{\mathrm{d}}=\mathrm{P}_{c} / 2$ or $\mathrm{P}_{c}=\mathrm{P}_{\mathrm{d}} / 2$ there is a maximum increase of $\mathrm{P}_{\text {eff }}$ at $\hat{\phi}_{d}=0$ or $\hat{\phi}_{d}=1$. In any case, this inflexion point cannot appear within the range $1 / 3<\hat{\phi}_{d}<2 / 3$. Note that according to the Levy model this inflexion would appear at $\hat{\phi}_{d}=1 / 2$. This is the justification to our attribution of an excessive symmetry to this model that, nevertheless, is the only model along with the EMA model to be able to predict an inflexion point.

Figure 3

In Figure 4 the $\mathrm{O}_{2}, \mathrm{~N}_{2}$ and $\mathrm{CH}_{4}$ permeabilities of the copolyimide BPDA-ODA$\operatorname{PEO}(2000)$ thermally treated at $250^{\circ} \mathrm{C}$ as a function of the volume fraction of PEO $\left(\phi_{d}\right)$ are depicted. The same features as seen in Figure 2 are now observed and in this case only the predictions of the EMA model are shown.

Figure 4

In Figure 5 the permeability for $\mathrm{O}_{2}$ of the copolyimide BPDA-ODA-PEO(2000) thermally treated at $250{ }^{\circ} \mathrm{C}$ is shown as a function of the volume fraction of PEO $\left(\phi_{\mathrm{d}}\right)$ along with the predictions of the models given by Equations: (3) (Brugeman) ; (4) (Bötcher); (5) (De Loor); (6) and (7) (Lewis-Nielsen) and by Equation (8) (Pal). The EMA model predictions, already shown in Figure 4 are drawn too for the sake of comparison. It seems clear that all these models diverge or at least do not approximate to the pure PEO permeability for $\phi_{\mathrm{d}}=1$, while the EMA model clearly succeeds to approach gradually the pure PEO permeability. The Lewis-Nielsen (with $\mathrm{A}=2$ ) and Pal (with $\mathrm{d}=3$ ) models have been taken with $\bar{\phi}_{d}=0.64$ as should correspond to the maximum packing of spherical inclusions. It seems clear that none of them adequately fits the experimental results probably because their application domain restrict to low content of the filler within a continuous matrix. 
In Figure 6 the values of $\bar{\phi}_{d}$ for the Pal model (that seems to be the most acceptable among the models shown in Figure 5) are changed in order to compare its predictions with the data for the $\mathrm{N}_{2}$ permeability (again for the BPDA-ODA-PEO(2000) copolyimide thermally treated at $250^{\circ} \mathrm{C}$ ) as a function of the volume fraction of PEO $\left(\phi_{\mathrm{d}}\right)$. It appears clear that the Pal model cannot account for the real dependence of permeability for any value of $\bar{\phi}_{d}$.

\section{Figure 6}

The predictions of the percolation model of Zhang (Equation (22)) clearly do not fit the experimental results for low volume fractions of PEO as shown for the $\mathrm{CH}_{4}$ permeability (for the BPDA-ODA-PEO(2000) copolyimide thermally treated at $250{ }^{\circ} \mathrm{C}$ ) in Figure 7. In this Figure, the two discontinuous lines correspond to fixed and fitted values for $\phi_{\mathrm{t}}$ and $\mathrm{P}_{\mathrm{t}}$. Clearly they do not show significant differences. The values obtained for $\mathrm{N}$ are also quite similar. It may be noted that the percolation based model proposed by Zhang et al. predicts that a percolation threshold would appear only if the ratio of the permeability of the filler to the polymer permeability was larger than $10^{5}$, which in the present case is not satisfied [62]. This requirement is clear in Figure 7 where it is seen that the assumption of a percolation threshold would lead to a predicted permeability of $\mathrm{CH}_{4}$ for the continuous phase that would be $10^{-5}$ barrer for a PEO permeability of the order of unity.

\section{Figure 7}

The values of N, 2.55 and 2.83, are somehow out of the range that could be expected for electrical or thermal conductivity and for permeability as far as it is well known that $\mathrm{N}$ $=2$ in a three-dimensional percolation (with an aspect ratio, $\delta=1$ ) [63], with $\delta=\mathrm{L} / \mathrm{D}, \mathrm{L}$ and $\mathrm{D}$ being the length and the diameter of the inclusions. 
In Figure 8 the EMA model is applied to the $\mathrm{CO}_{2}$ permeability of the copolyimides: BPDA-ODA-PEO(2000), BKDA-ODA-PEO(2000) and PMDA-ODA-PEO(2000). The figure shows that a change in the dianhydride has little effect in the dependence of permeability and the point of maximal increase in the effective permeability is quite similar and very close to a volume fraction of PEO $\hat{\phi}_{d} \approx 1 / 3$. This could be predicted from Equations (23) and (24) because $\lambda$ goes from $2.8 \cdot 10^{-3}$ to $17.4 \cdot 10^{-3}$ which corresponds to values of $\hat{\phi}_{d}$ going from $32.7 \%$ to $33.2 \%$.

\section{Figure 8}

Figure 9 and 10 correspond to a three phase system BKDA-ODA-PEO(6000) constituted by the pure BKDA-ODA, the amorphous $\operatorname{PEO}(6000)$ and the crystalline one. In Figure 9 the white symbols correspond to the total volume proportion of PEO(6000). The EMA model fits the results only when the actual amorphous volume proportion of $\mathrm{PEO}(6000)$ is taken into account after subtraction of the crystalline volume as obtained by DSC, [16], and the system is taken as a bi-phase system.

In Figure 10 the three phase system has been fitted to Equation (10), for three phases:

$$
\left.\begin{array}{c}
\sum_{i=1}^{3} \phi_{i} \frac{P_{i}-P_{e f f}}{P_{i}+2 P_{e f f}}=0 \\
\sum_{i=1}^{3} \phi_{i}=1
\end{array}\right\}
$$

assuming that the permeability of the crystalline PEO is zero. It is clear that as shown in Figure 10, the EMA model can predict also the permeability across three phase composite segregated copolyimides. The graph corresponds to the effective permeability for the mentioned ternary system. In the Figure, the composition of the ternary system is given in terms of the volume fractions of crystalline and amorphous PEO. The datum for the semicrystalline PEO with $29 \%$ crystallinity has been taken from Lin and Freeman [64] (see Figure 2 of this reference).

\section{Figure 9}




\section{Conclusions}

A series of aliphatic aromatic copoly(etherimide)s has been synthesized. Cast films of these copolymers have been thermally treated to ensure the phase segregation of the linear PEO chains from the aromatic moiety of the copoly(etherimide)s. The permeability of $\mathrm{O}_{2}, \mathrm{~N}_{2}, \mathrm{CO}_{2}$ and $\mathrm{CH}_{4}$ of the resulting membranes have been compared with the predictions of the main usable models adapted to the gas permeability.

The model based on the Effective Medium Approximation (EMA) succeeded to predict the main features of the experimental results. Specifically, it was able to calculate the volume fraction for the maximum increase of permeability, a common feature for all the studied segregated copolymer membranes. The model was even able to predict the permeabilities of a three phase system consisting in the aromatic (BKDA-ODA) rich phase plus the amorphous $\mathrm{PEO}(6000)$ and the crystalline $\mathrm{PEO}(6000)$ phases.

As discussed, the EMA calculations are especially applicable to composites and polycrystals in which the various components are randomly and symmetrically distributed, so that none of the components is identifiable as a host in which the others are preferentially embedded. The size and orientation of each phase are continuously changing with their proportion; this is the case with the segregated copoly(ether)imides studied here. On the other hand, there is not a constant size network to be filled and the permeabilities of the phases involved are relatively close to each other as to make percolation impossible. Nevertheless they are different enough to have a certain percolation-like sudden step in the effective permeability which is well integrated in the EMA model.

The model has the advantage of being able to be adapted for three phase (or multiphase) composites where the structure of each phase in the mixture is effectively random in nature. This fact has the added advantage of making unnecessary any consideration on the detailed phase-to-phase morphology. 


\section{Aknowledgements}

The authors thank the Ministerio de Educación y Ciencia (Plan Nacional de I+D+i) through projects MAT2011-25513 and CTQ2012-31076, Junta de Castilla y León (project VA-324A11-2) and also Acciona Agua for partial funding of this research. We are indebted also to Ministerio de Economía y Competitividad through project MAT2010-20668. A. Tena thanks CSIC for a predoctoral JAE fellowship.

\section{References}

[1] R.W. Baker, Future directions of membrane gas separation technology, Industrial \& Engineering Chemistry Research, 41 (2002) 1393-1411.

[2] R. Bounaceur, N. Lape, D. Roizard, C. Vallieres, E. Favre, Membrane processes for post-combustion carbon dioxide capture: A parametric study, Energy, 31 (2006) 25562570.

[3] E. Favre, Carbon dioxide recovery from post-combustion processes: Can gas permeation membranes compete with absorption?, Journal of Membrane Science, 294 (2007) 50-59.

[4] I. Bessonov, W.W. Wright, Polyimides: thermally stable polymers, Consultants Bureau, 1987.

[5] M.K. Ghosh, K.L. Mittal, Polyimides: Fundamentals and Applications, Marcel Dekker Incorporated, 1996.

[6] K.L. Mittal, Polyimides and Other High-temperature Polymers: Synthesis, Characterization, and Applications. Volume 5, VSP, 2009.

[7] K. Tanaka, H. Kita, M. Okano, K.-i. Okamoto, Permeability and permselectivity of gases in fluorinated and non-fluorinated polyimides, Polymer, 33 (1992) 585-592.

[8] D. Ayala, A. Lozano, J. De Abajo, C. Garcia-Perez, J. De la Campa, K.-V. Peinemann, B. Freeman, R. Prabhakar, Gas separation properties of aromatic polyimides, Journal of Membrane Science, 215 (2003) 61-73.

[9] K. Tanaka, H. Kita, K. Okamoto, A. Nakamura, Y. Kusuki, Gas permeability and permselectivity in polyimides based on 3, 3', 4, 4'-biphenyltetracarboxylic dianhydride, Journal of Membrane Science, 47 (1989) 203-215.

[10] T. Barbari, W. Koros, D. Paul, Gas transport in polymers based on bisphenol-A, Journal of Polymer Science Part B: Polymer Physics, 26 (1988) 709-727. 
[11] Y. Li, M. Ding, J. Xu, Gas separation properties of aromatic polyetherimides from its esters, Journal of applied polymer science, 63 (1997) 1-7.

[12] A. Tena, A. Marcos-Fernández, A.E. Lozano, J.G. de la Campa, J. de Abajo, L. Palacio, P. Prádanos, A. Hernández, Thermally treated copoly (ether-imide) s made from bpda and alifatic plus aromatic diamines. Gas separation properties with different aromatic diamimes, Journal of Membrane Science, 387 (2012) 54-65.

[13] A. Marcos-Fernández, A. Tena, A.E. Lozano, J.G. de la Campa, J. de Abajo, L. Palacio, P. Prádanos, A. Hernández, Physical properties of films made of copoly (etherimide) s with long poly (ethylene oxide) segments, European Polymer Journal, 46 (2010) 2352-2364.

[14] A. Gil, A. Tena, A. Marcos-Fernández, M.R. De La Viuda, A.E. Lozano, P. Prádanos, Block copolymers for gas separation: Looking for the ideal structure, in: Elsevier (Ed.) Third International Symposium: Frontiers in Polymer Science, Sitges (Spain), 2013.

[15] A. Tena, A. Marcos-Fernández, L. Palacio, P. Cuadrado, P. Prádanos, J. de Abajo, A.E. Lozano, A. Hernández, Phase Segregation and Gas Separation Properties of Thermally Treated Copoly (ether-imide) from an Aromatic Dianhydride, an Aromatic Diamine, and Various Aliphatic Diamines, Industrial \& Engineering Chemistry Research, 51 (2012) 3766-3775.

[16] A. Tena, A. Lozano, L. Palacio, A. Marcos-Fernández, P. Prádanos, J. de Abajo, A. Hernández, Gas separation properties of systems with different amounts of long poly (ethylene oxide) segments for mixtures including carbon dioxide, International Journal of Greenhouse Gas Control, 12 (2013) 146-154.

[17] A. Tena, A. Marcos-Fernandez, A.E. Lozano, J.G. De La Campa, J. De Abajo, L. Palacio, P. Pradanos, A. Hernandez, Thermally Segregated Copolymers with PPO Blocks for Nitrogen Removal from Natural Gas, Industrial \& Engineering Chemistry Research, (2013).

[18] Song Lin Liu, Lu Shao, Mei Ling Chua, Cher Hon Lau, Huan Wang, Shuai Quan, "Recent progress in the design of advanced PEO-containing membranes for CO2 removal" Progress in Polymer Science, 38(7) (2013) 1089-1120.

[19] A. Tena, A. Marcos-Fernández, L. Palacio, P. Prádanos, A.E. Lozano, J.d. Abajo, A. Hernández, On the influence of the proportion of PEO in thermally controlled phase segregation of copoly(ether-imide)s for gas separation, Journal of Membrane Science, (2013).

[20] H. Chen, Y. Xiao, T.-S. Chung, Synthesis and characterization of poly (ethylene oxide) containing copolyimides for hydrogen purification, Polymer, 51 (2010) 40774086. 
[21] Metz SJ, Mulder MHV, Wessling M. Gas-permeation properties of poly(ethyleneoxide) poly(butylene terephthalate) block copolymers, Macromolecules 237 (2004)4590-4597.

[22] A. Dufresne, J.-Y. Cavaille, Clustering and percolation effects in microcrystalline starch-reinforced thermoplastic, Journal of Polymer Science Part B Polymer Physics, 36 (1998) 2211-2224.

[23] O. Levy, D. Stroud, Maxwell Garnett theory for mixtures of anisotropic inclusions: Application to conducting polymers, Physical Review B, 56 (1997) 8035.

[24] M. Zimmer, X. Fan, J. Bao, R. Liang, B. Wang, C. Zhang, J. Brooks, ThroughThickness Thermal Conductivity Prediction Study on Nanocomposites and Multiscale Composites, Materials Sciences and Applications, 3 (2012) 131-138.

[25] B. Shimekit, H. Mukhtar, Gas permeation models in mixed matrix membranes, in: National Postgraduate Conference (NPC), 2011, IEEE, 2011, pp. 1-5.

[26] J.M. Garnett, Colours in metal glasses, in metallic films, and in metallic solutions. II, Philosophical Transactions of the Royal Society of London. Series A, Containing Papers of a Mathematical or Physical Character, (1906) 237-288.

[27] A. Eucken, Allgemeine gesetzmabigkeiten fur das warmeleitvermogen verschiedener stoffarten and aggregatzustande, Forschung Gabiete Ingenieur, 11 (1940) $6-20$.

[28] D.J. Bergman, D. Stroud, Physical properties of macroscopically inhomogeneous media, Solid State Physics, 46 (1992) 147-269.

[29] J.C. Garland, D.B. Tanner, O.S. University, U.S.E. Research, D. Administration, N.S. Foundation, Electrical transport and optical properties of inhomogeneous media, Ohio State University, 1977, American Institute of Physics, 1978.

[30] D.A.G. Bruggeman, Calculation of various physical constants in heterogeneous substances. I. Dielectric constants and conductivity of composites from isotropic substances, Ann. Phys. (Paris), 24 (1935) 636.

[31] M. Haddadi, B. Agoudjil, A. Boudenne, B. Garnier, Analytical and Numerical Investigation on Effective Thermal Conductivity of Polymer Composites Filled with Conductive Hollow Particles, International Journal of Thermophysics, 34 (2013) 101112.

[32] C.J.F. Böttcher, Theory of electric polarisation, Elsevier Pub. Co., Amsterdam, the Netherlands, 1952.

[33] G. De Loor, Dielectric properties of heterogeneous mixtures, University of Leiden, Leiden, Uitgeverij Excelsior, 1956. 
[34] H. Ebadi-Dehaghani, M. Nazempour, Thermal Conductivity of Nanoparticles Filled Polymers, in: D.A. Hashim (Ed.) Nanotechnology and Nanomaterials: "Smart Nanoparticles Technology", InTech, 2012.

[35] T. Lewis, L. Nielsen, Dynamic mechanical properties of particulate-filled composites, Journal of applied polymer science, 14 (1970) 1449-1471.

[36] L.E. Nielsen, Thermal conductivity of particulate filled polymers, Journal of applied polymer science, 17 (1973) 3819-3820.

[37] L.E. Nielsen, The thermal and electrical conductivity of two-phase systems, Industrial \& Engineering Chemistry Fundamentals, 13 (1974) 17-20.

[38] D. Kumlutaş, I.H. Tavman, M. Turhan Çoban, Thermal conductivity of particle filled polyethylene composite materials, Composites Science and Technology, 63 (2003) 113-117.

[39] B. Weidenfeller, M. Höfer, F.R. Schilling, Thermal conductivity, thermal diffusivity, and specific heat capacity of particle filled polypropylene, Composites Part A: applied science and manufacturing, 35 (2004) 423-429.

[40] D. Kumlutaş, I.H. Tavman, A numerical and experimental study on thermal conductivity of particle filled polymer composites, Journal of Thermoplastic Composite Materials, 19 (2006) 441-455.

[41] R. Pal, On the Lewis-Nielsen model for thermal/electrical conductivity of composites, Composites Part A: applied science and manufacturing, 39 (2008) 718-726.

[42] H. Davis, L. Valencourt, C. Johnson, Transport processes in composite media, Journal of the American Ceramic Society, 58 (1975) 446-452.

[43] R. Landauer, The electrical resistance of binary metallic mixtures, Journal of Applied Physics, 23 (1952) 779-784.

[44] P. Karayacoubian, Effective thermal conductivity of composite fluidic thermal interface materials, in: Applied Science In Mechanical Engineering, Univ. Waterloo, Ontario, Canada 2006.

[45] X. Colin Tong Advanced Materials for Thermal Management of Electronic Packaging, Sprienger series in advanced microelectronics 30, Springer Science+Business Media, New York, USA, 2011.

[46] D.L. Johnson, P.N. Sen, Physics and chemistry of porous media, American Institute of Physics, 1984.

[47] F. Levy, A modified Maxwell-Eucken equation for calculating the thermal conductivity of two-component solutions or mixtures, International Journal of Refrigeration, 4 (1981) 223-225. 
[48] J. Wang, J.K. Carson, M.F. North, D.J. Cleland, A new approach to modelling the effective thermal conductivity of heterogeneous materials, International Journal of Heat and Mass Transfer, 49 (2006) 3075-3083.

[49] T.-S. Chung, L.Y. Jiang, Y. Li, S. Kulprathipanja, Mixed matrix membranes (MMMs) comprising organic polymers with dispersed inorganic fillers for gas separation, Progress in Polymer Science, 32 (2007) 483-507.

[50] R. Mahajan, W.J. Koros, Mixed matrix membrane materials with glassy polymers. Part 1, Polymer Engineering \& Science, 42 (2002) 1420-1431.

[51] T.T. Moore, R. Mahajan, D.Q. Vu, W.J. Koros, Hybrid membrane materials comprising organic polymers with rigid dispersed phases, AIChE journal, 50 (2004) 311-321.

[52] J. Felske, Effective thermal conductivity of composite spheres in a continuous medium with contact resistance, International Journal of Heat and Mass Transfer, 47 (2004) 3453-3461.

[53] B. Shimekit, H. Mukhtar, T. Murugesan, Prediction of the relative permeability of gases in mixed matrix membranes, Journal of Membrane Science, 373 (2011) 152-159.

[54] S.R. Broadbent, J.M. Hammersley, Percolation processes I. Crystals and mazes, in: Proc. Cambridge Philos. Soc, 1957, pp. 629-641.

[55] G. Grimmet, Percolation, vol. 321 in A Series of Comprehensive Studies in Mathematics, Springer, Berlin, Germany, 1999.

[56] D. Bigg, The effect of compounding on the conductive properties of EMI shielding compounds, Advances in Polymer Technology, 4 (1984) 255-266.

[57] J. Clerc, G. Giraud, J. Laugier, J. Luck, The electrical conductivity of binary disordered systems, percolation clusters, fractals and related models, Advances in Physics, 39 (1990) 191-309.

[58] Y.P. Mamunya, V. Davydenko, P. Pissis, E. Lebedev, Electrical and thermal conductivity of polymers filled with metal powders, European Polymer Journal, 38 (2002) 1887-1897.

[59] W. Tian, R. Yang, Phonon transport and thermal conductivity percolation in random nanoparticle composites, Computer modeling in engineering and sciences, 24 (2008) 123.

[60] I.U.K. Godovskiı̌, V.P. Privalko, Thermal and electrical conductivity of polymer materials, Springer, New York, USA, 1995.

[61] G. Zhang, Y. Xia, H. Wang, Y. Tao, G. Tao, S. Tu, H. Wu, A percolation model of thermal conductivity for filled polymer composites, Journal of composite materials, 44 (2010) 963-970. 
[62] M. Kole, D. Tripathi, T.K. Dey, Percolation based enhancement in effective thermal conductivity of HDPE/LBSMO composites, Bulletin of Materials Science, 35 (2012) 601-609.

[63] D. Stauffer, A. Aharony, Introduction to Percolation Theory, Taylor \& Francis Group, London. UK, 1994.

[64] H. Lin, B.D. Freeman, Gas solubility, diffusivity and permeability in poly (ethylene oxide), Journal of Membrane Science, 239 (2004) 105-117. 
Figure 1.- Structure of the aromatic and aliphatic components of the copolyimide thermally segregated membranes studied here along with their densities. $\left(^{a}\right)$ Data from the manufacturer and $\left({ }^{b}\right)$ Tanaka et al. [7]. Volume fractions $\phi_{d}$ can be evaluated from weight fractions $\omega_{d}$ by: $\phi_{d}=\left(\omega_{d} / \rho_{d}\right) /\left[\left(\omega_{d} / \rho_{d}\right)+\left(1-\omega_{d}\right) / \rho_{c}\right]$. $\rho_{d}$ is the density of filler and $\rho_{c}$ is the density of the matrix.

Figure 2.- Comparison of some models for the effective permeability of $\mathrm{CO}_{2}$ for a maximum thermally segregated polyimide. The Bruggeman model corresponds to Equation (2) with $d=3$. The EMA model corresponds to the s-shaped continuous thick line.

Figure 3.- Plot of Equation (1724) showing that there is a range from $\phi_{\mathrm{d}}=1 / 3$ to $2 / 3$ that can't correspond to the inflexion point in $\mathrm{P}$ eff. The inflexion point for the Levy model should be placed in $\phi_{\mathrm{d}}=1 / 2$.

Figure 4.- Effective permeability of: $\mathrm{O}_{2}, \mathrm{~N}_{2}$ and $\mathrm{CH}_{4}$ for a thermally segregated polyimide. The lines correspond to the EMA model. Vertical lines correspond to the maximum increase in permeability.

Figure 5.- Effective permeability of $\mathrm{O}_{2}$ and predictions of some models for low content of a dispersed phase. The Lewis-Nielsen $(A=2)$ and the Pal $(d=3)$ models have been shown for $\bar{\phi}_{d}=0.64$. The EMA model predictions are shown for the sake of comparison. Vertical lines correspond to the maximum increase in permeability

Figure 6.- Effective permeability of $\mathrm{N}_{2}$ and predictions of the Pal model (with $\mathrm{d}=3$ ) for three different $\bar{\phi}_{d}$. The double vertical lines correspond to the maximum increase in permeability while the other vertical lines correspond to the asymptotes of the Pal model. 
Figure 7.- Effective permeability of $\mathrm{CH}_{4}$ and predictions of the percolation model of Zhang. The white cross corresponds to a fixed pair $\phi_{t}$ and $P_{t}$ while the gray one corresponds to values obtained for $\phi_{\mathrm{t}}$ and $\mathrm{P}_{\mathrm{t}}$ after fitting.

Figure 8.- Effective permeability of $\mathrm{CO}_{2}$ for thermally segregated polyimides with different dianhydrides. The lines correspond to the EMA model.

Figure 9.- Effective permeability of $\mathrm{CO}_{2}$ for a partially crystalline $\mathrm{PEO}$ within a BKDA-ODA matrix at $30^{\circ} \mathrm{C}(\mathrm{O})$. When the fractions of real amorphous PEO $(\oplus)$ are taken into account EMA predictions reproduce the experimental results. The line correspond to the EMA model. At $50^{\circ} \mathrm{C}(\mathbf{O})$ there is no crystallinity and the EMA predictions are again quite good.

Figure 10.- Effective permeability of $\mathrm{CO}_{2}$ for a three-phase system of crystalline and amorphous PEO in a BKDA-ODA matrix and the predictions of EMA. 


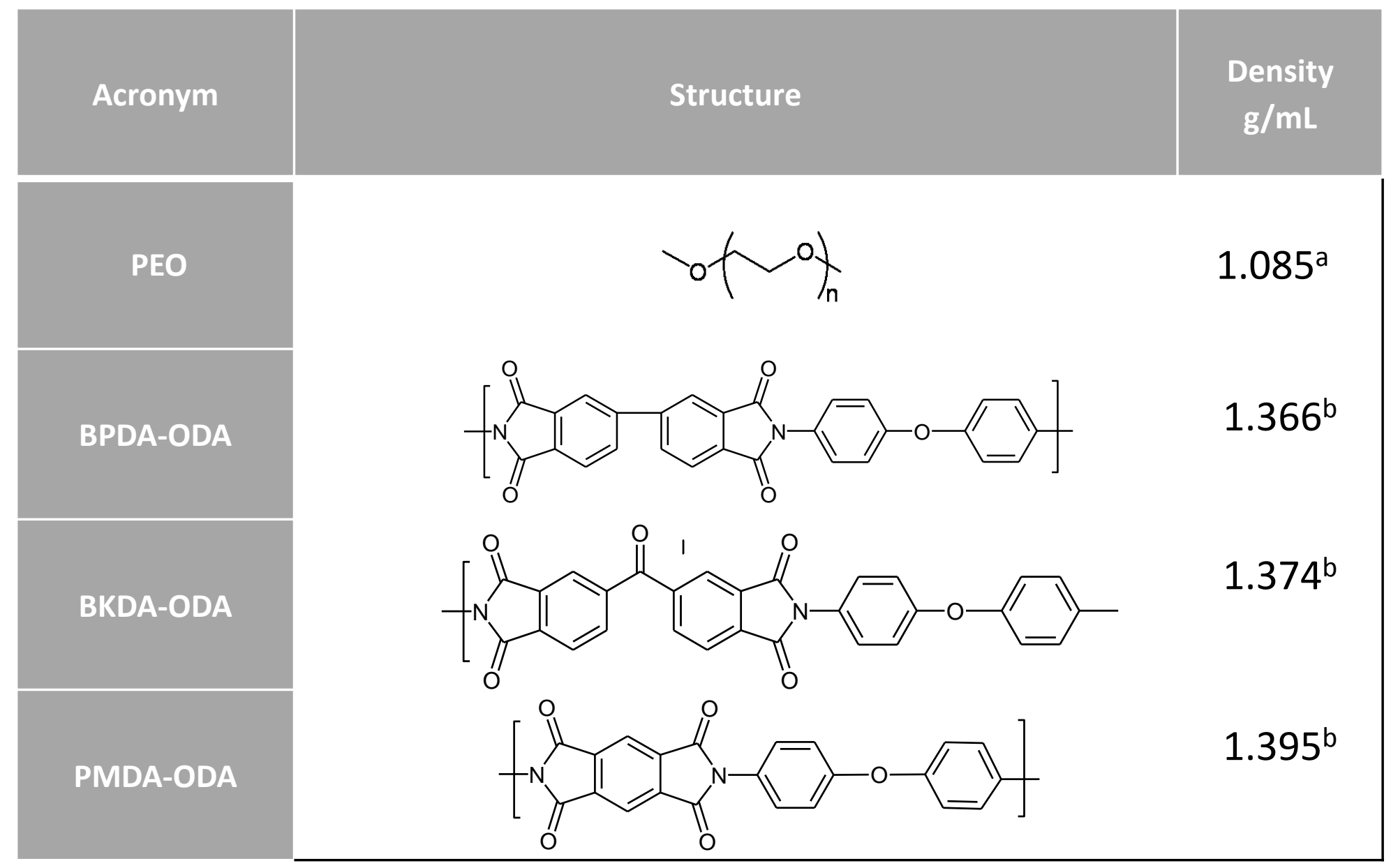

Figure 1.- Structure of the aromatic and aliphatic components of the copolyimide thermally segregated membranes studied here along with their densities. (a) Data from the manufacturer and (b) Tanaka et al. [7]. Volume fractions $\phi_{d}$ can be evaluated from weight fractions $\omega_{d}$ by: $\phi_{d}=$ $\left(\omega_{d} / \rho_{d}\right) /\left[\left(\omega_{d} / \rho_{d}\right)+\left(1-\omega_{d}\right) / \rho_{c}\right] . \rho_{d}$ is the density of filler and $\rho_{c}$ is the density of the matrix. 


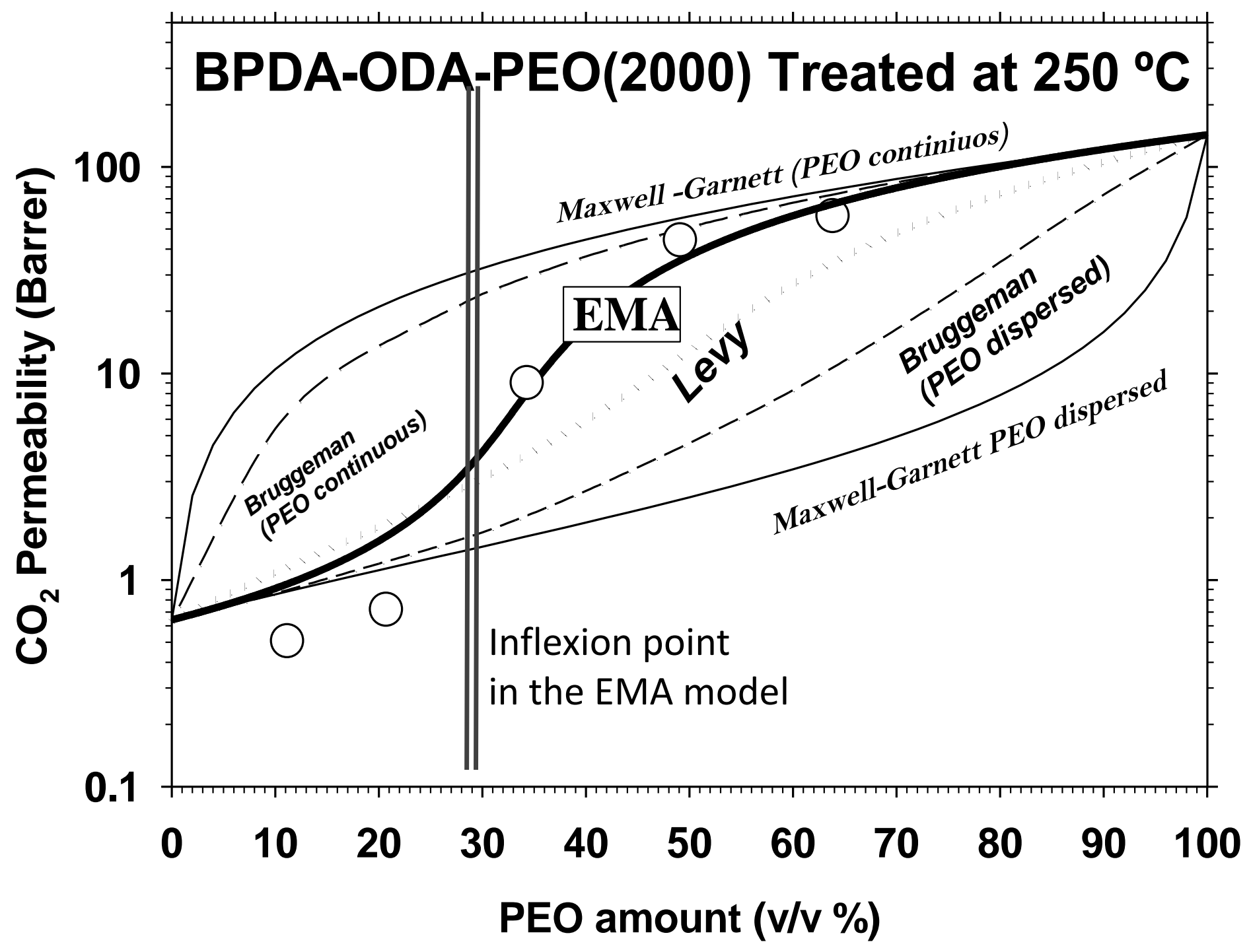

Figure 2.- Comparison of some models for the effective permeability of $\mathrm{CO}_{2}$ for a maximum thermally segregated polyimide. The Bruggeman model corresponds to Equation (2) with $d=3$. The EMA model corresponds to the s-shaped continuous thick line. 


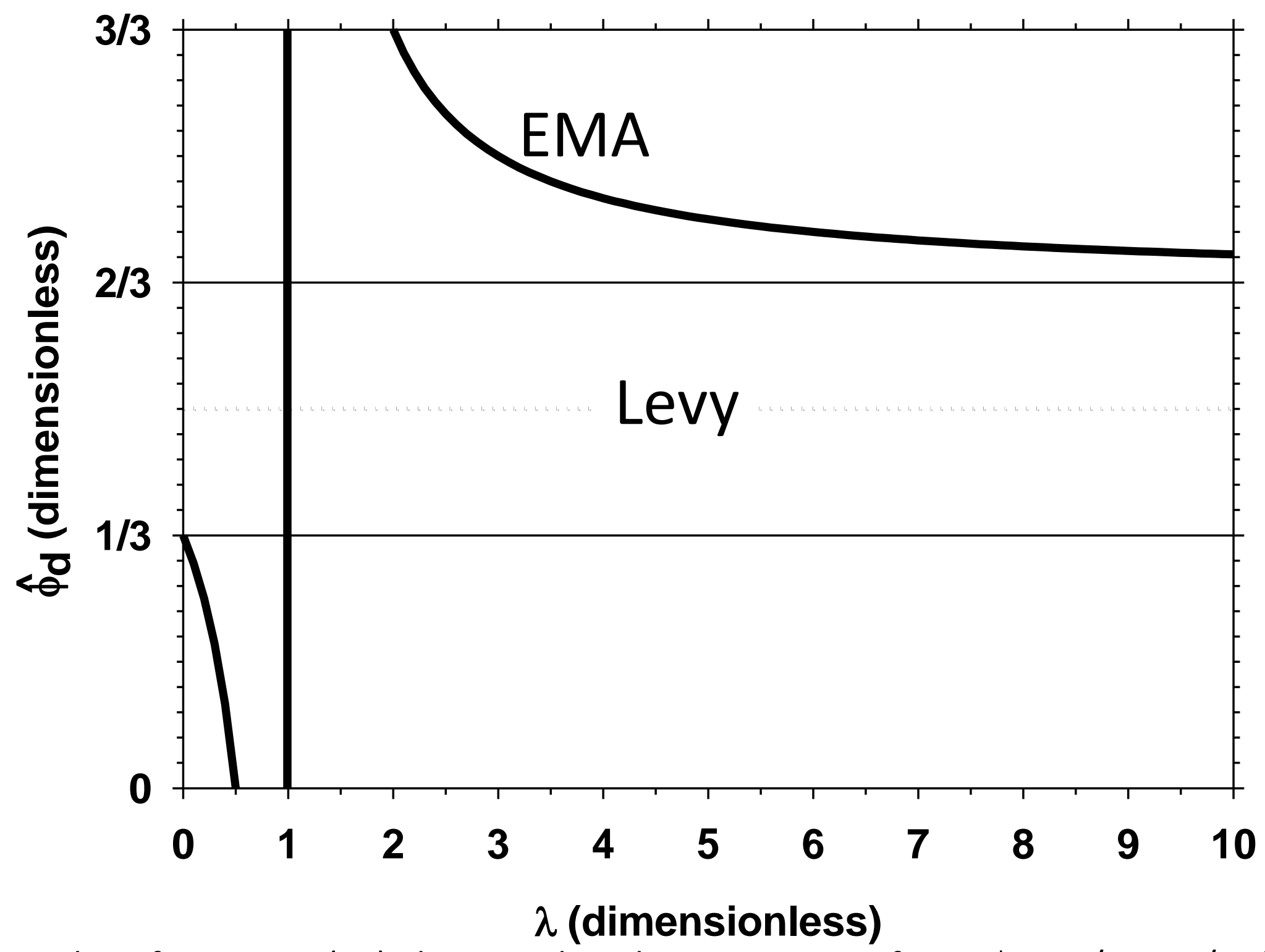

Figure 3.- Plot of Equation (17) showing that there is a range from $\phi_{d}=1 / 3$ to $2 / 3$ that can't correspond to the inflexion point in $\mathrm{P}_{\text {eff }}$. The inflexion point for the Levy model should be placed in $\phi_{d}=1 / 2$ 


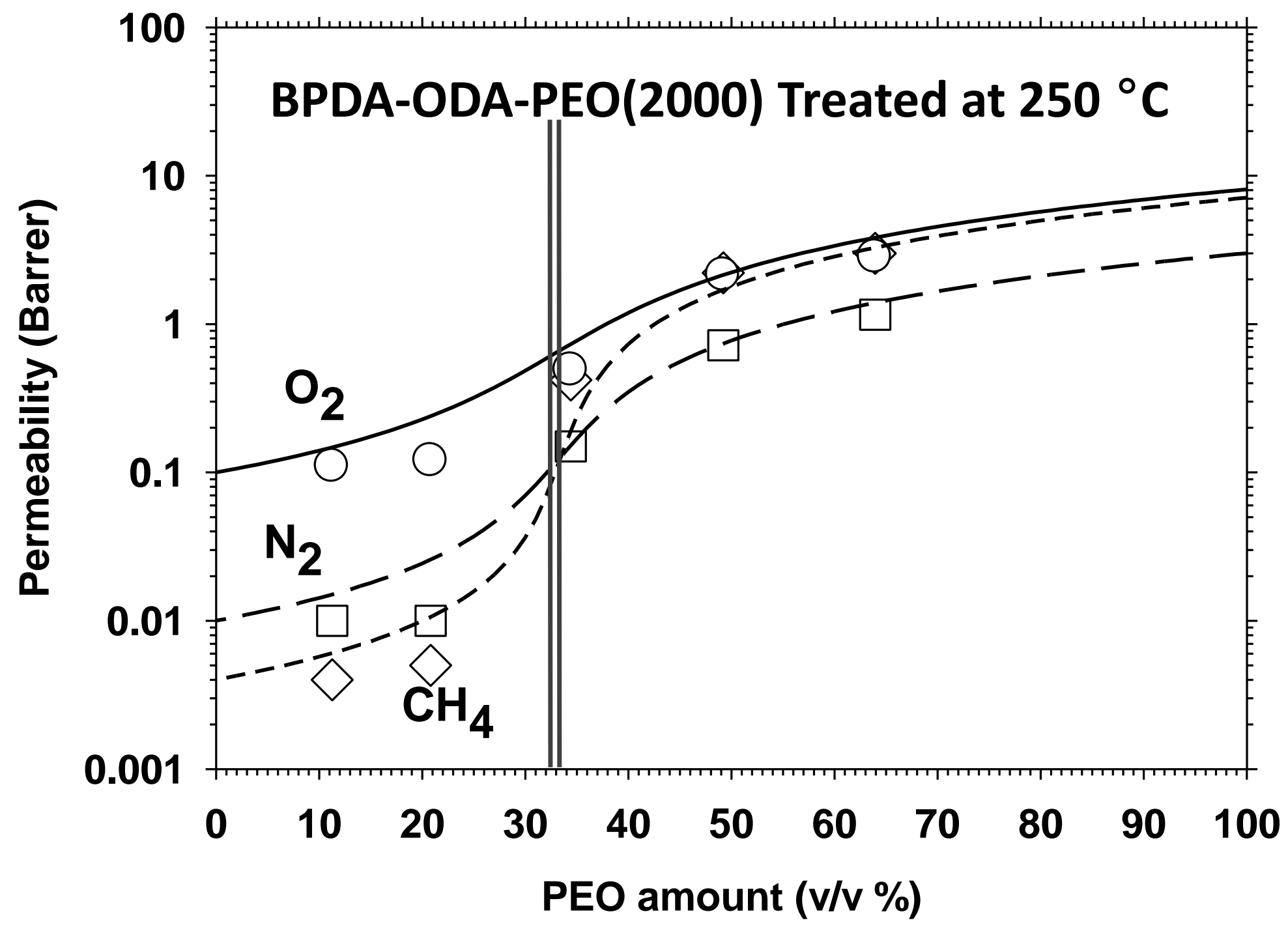

Figure 4.- Effective permeability of: $\mathrm{O}_{2}, \mathrm{~N}_{2}$ and $\mathrm{CH}_{4}$ for a thermally segregated polyimide. The lines correspond to the EMA model. Vertical lines correspond to the maximum increase in permeability. 


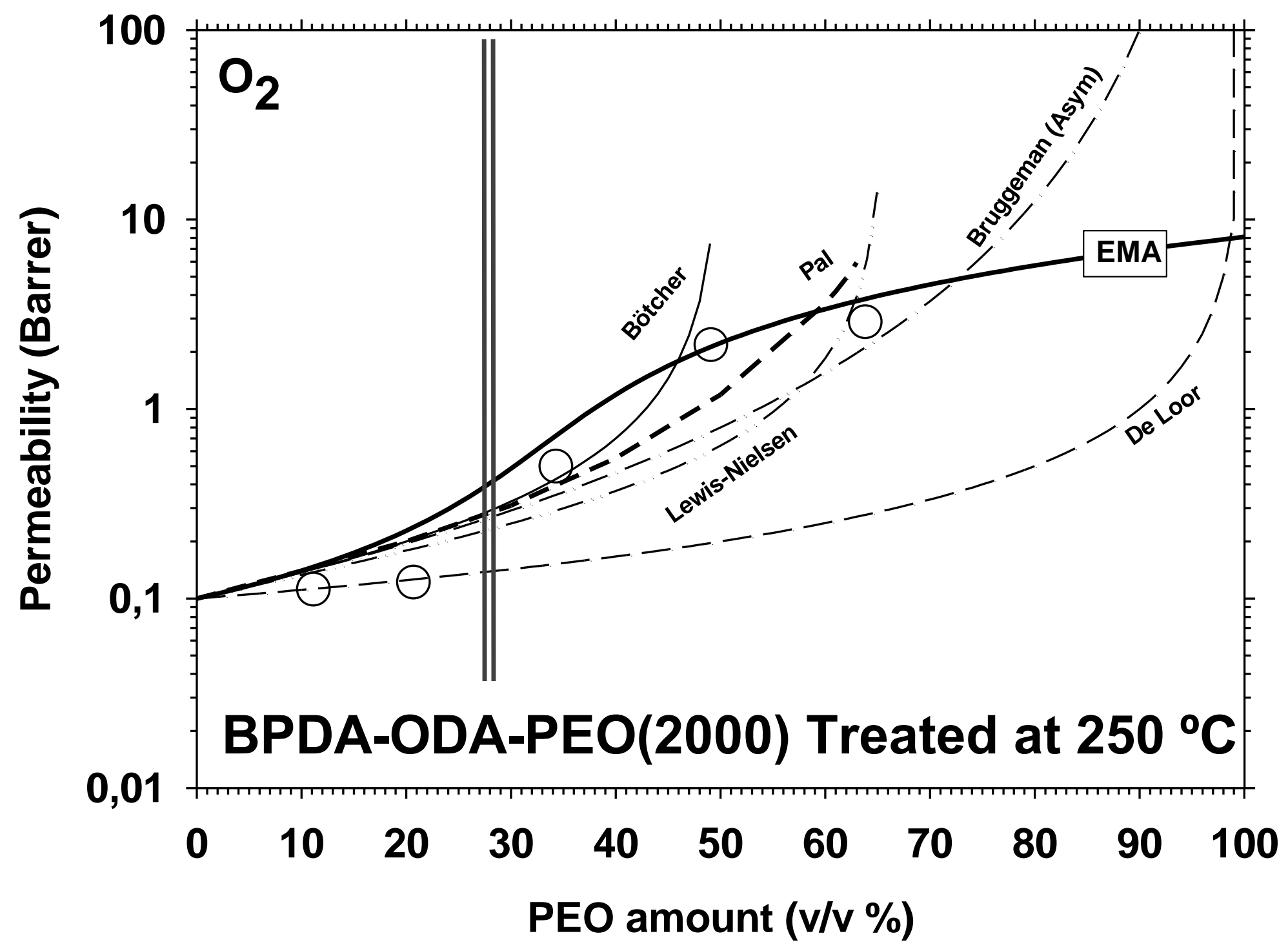

Figure 5.- Effective permeability of $\mathrm{O}_{2}$ and predictions of some models for low content of a dispersed phase. The Lewis-Nielsen (with $A=2$ ) and the Pal (with $d=3$ ) models have been shown for $\bar{\phi}_{d}=0.64$. The EMA model predictions are shown for the sake of comparison. Vertical lines correspond to the maximum increase in permeability. 


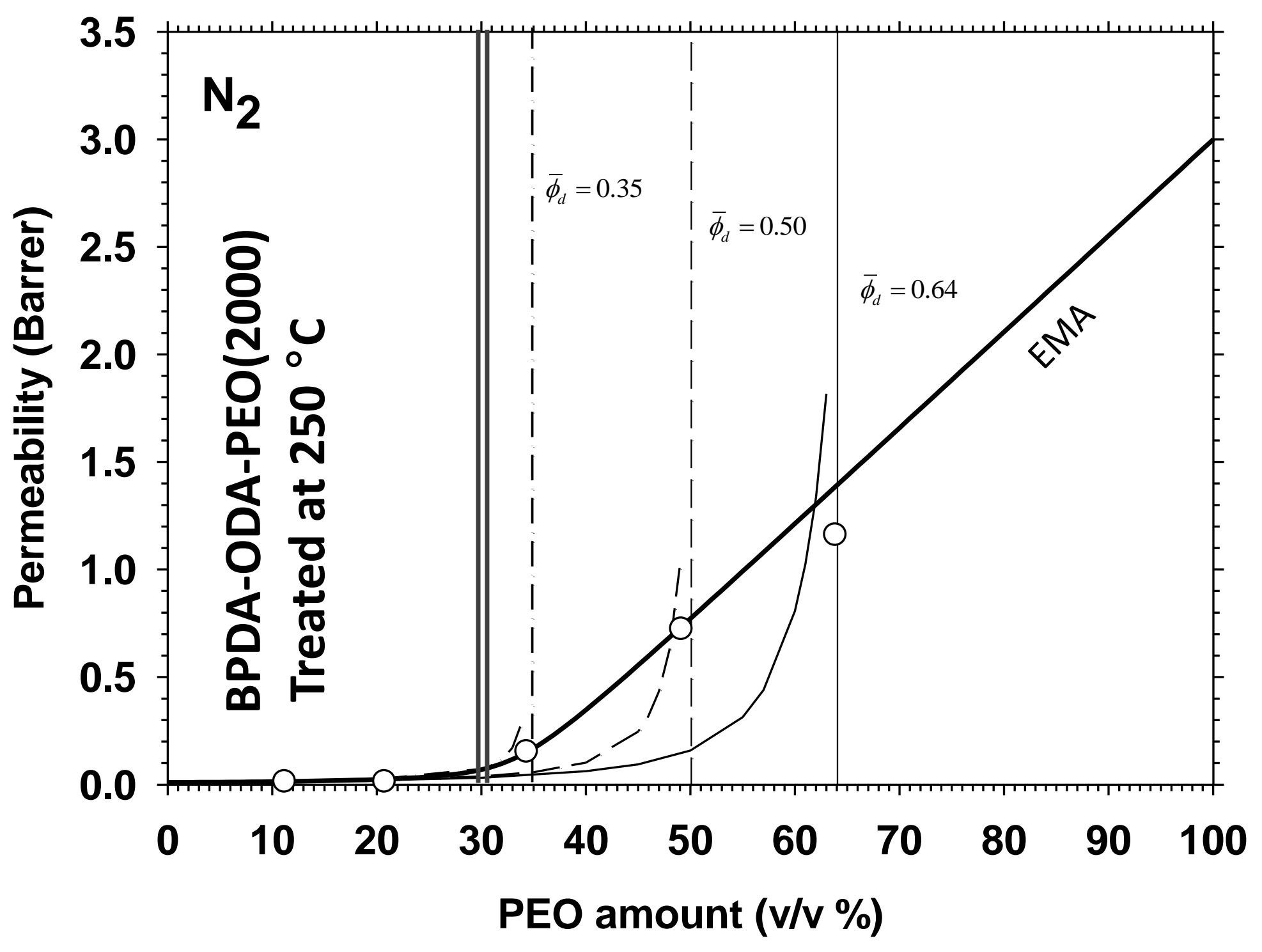

Figure 6.- Effective permeability of $\mathrm{N}_{2}$ and predictions of the Pal model (with $d=3$ ) for three different $\bar{\phi}_{d}$. The double vertical lines correspond to the maximum increase in permeability while the other vertical lines correspond to the asymptotes of the Pal model. 


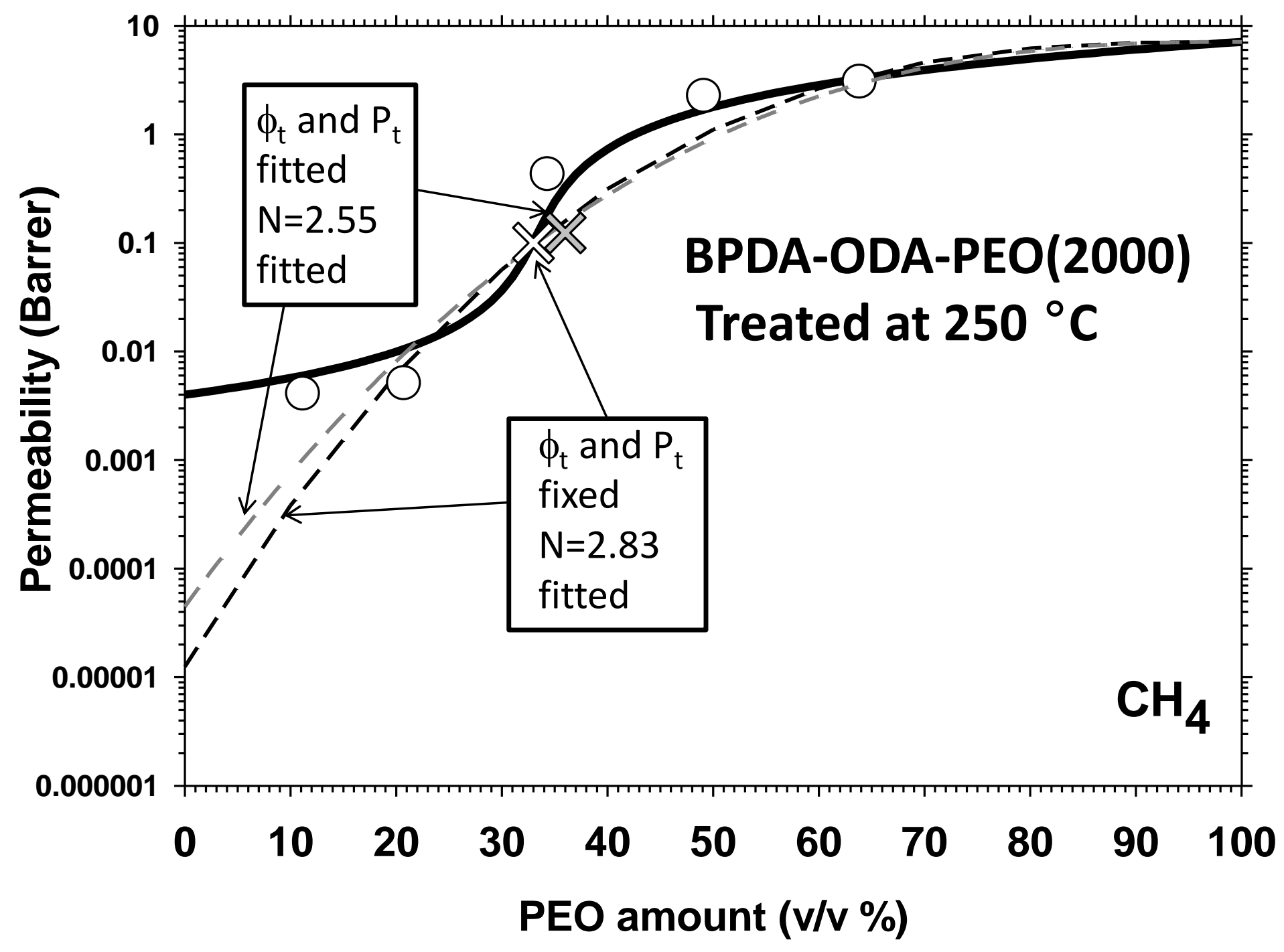

Figure 7.- Effective permeability of $\mathrm{CH}_{4}$ and predictions of the percolation model of Zhang. The white cross corresponds to a fixed pair $\phi_{t}$ and $P_{t}$ while the gray one corresponds to values obtained for $\phi_{t}$ and $P_{t}$ after fitting. 


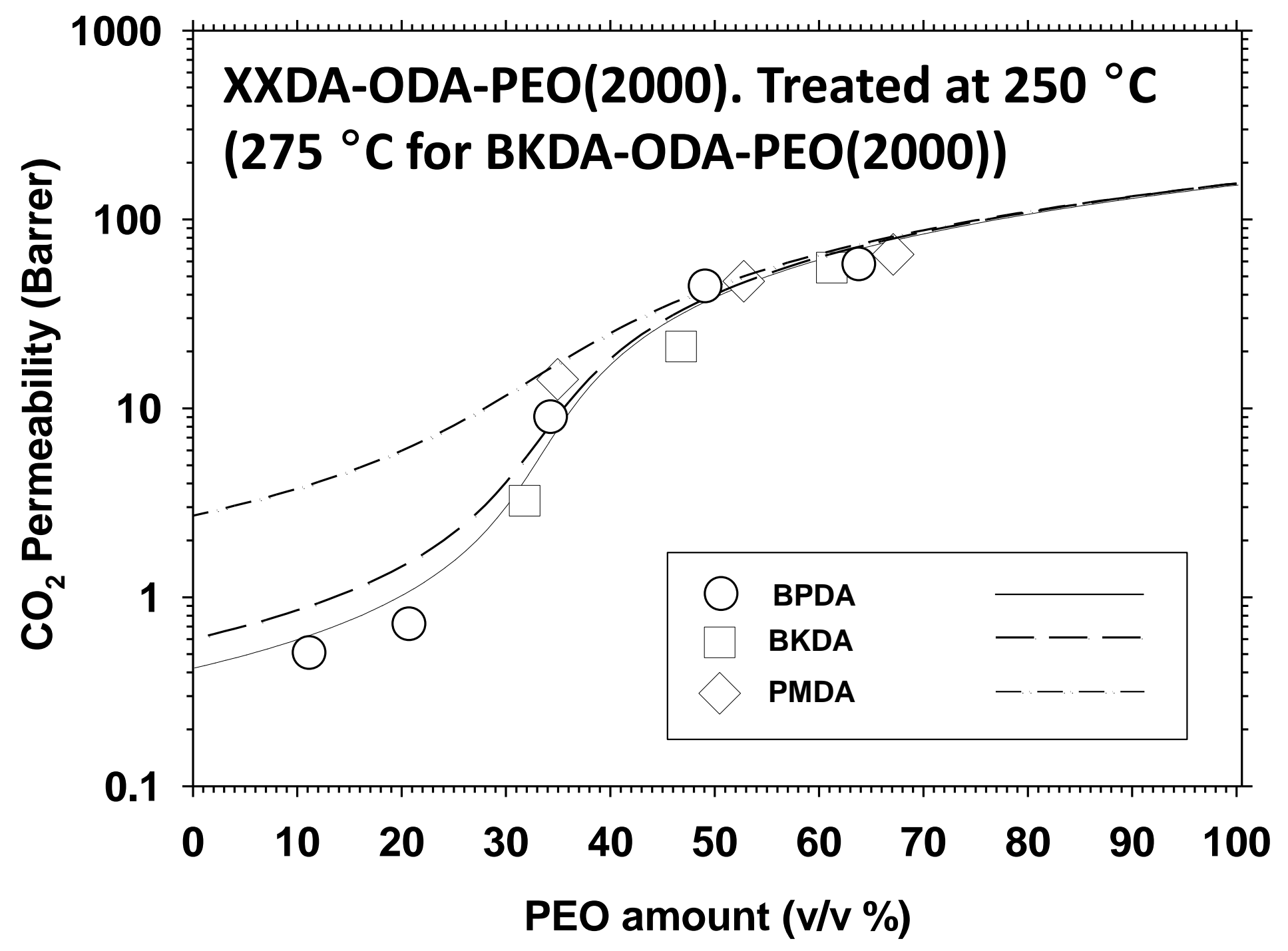

Figure 8.- Effective permeability of $\mathrm{CO}_{2}$ for thermally segregated polyimides with different dianhydrides. The lines correspond to the EMA model. 


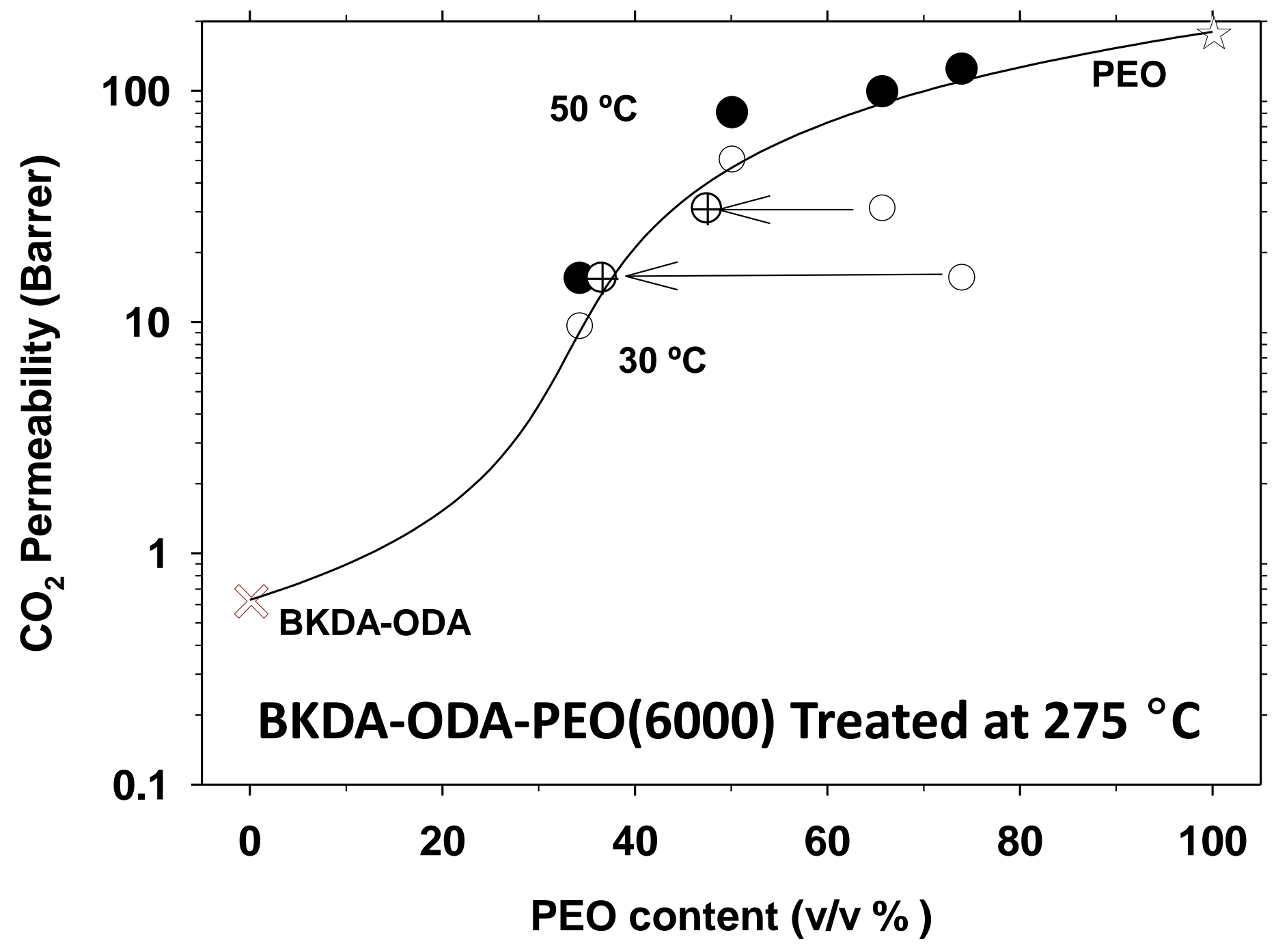

Figure 9.- Effective permeability of $\mathrm{CO}_{2}$ for a partially crystalline PEO within a BKDA-ODA matrix at $30 \stackrel{\circ}{ }{ }^{\circ}(O)$. When the fractions of real amorphous PEO $(\oplus)$ are taken into account EMA predictions reproduce the experimental results. The line correspond to the EMA model. At $50 \cong$ $\mathrm{C}(\mathbf{O})$ there is no crystallinity and the EMA predictions are again quite good. 


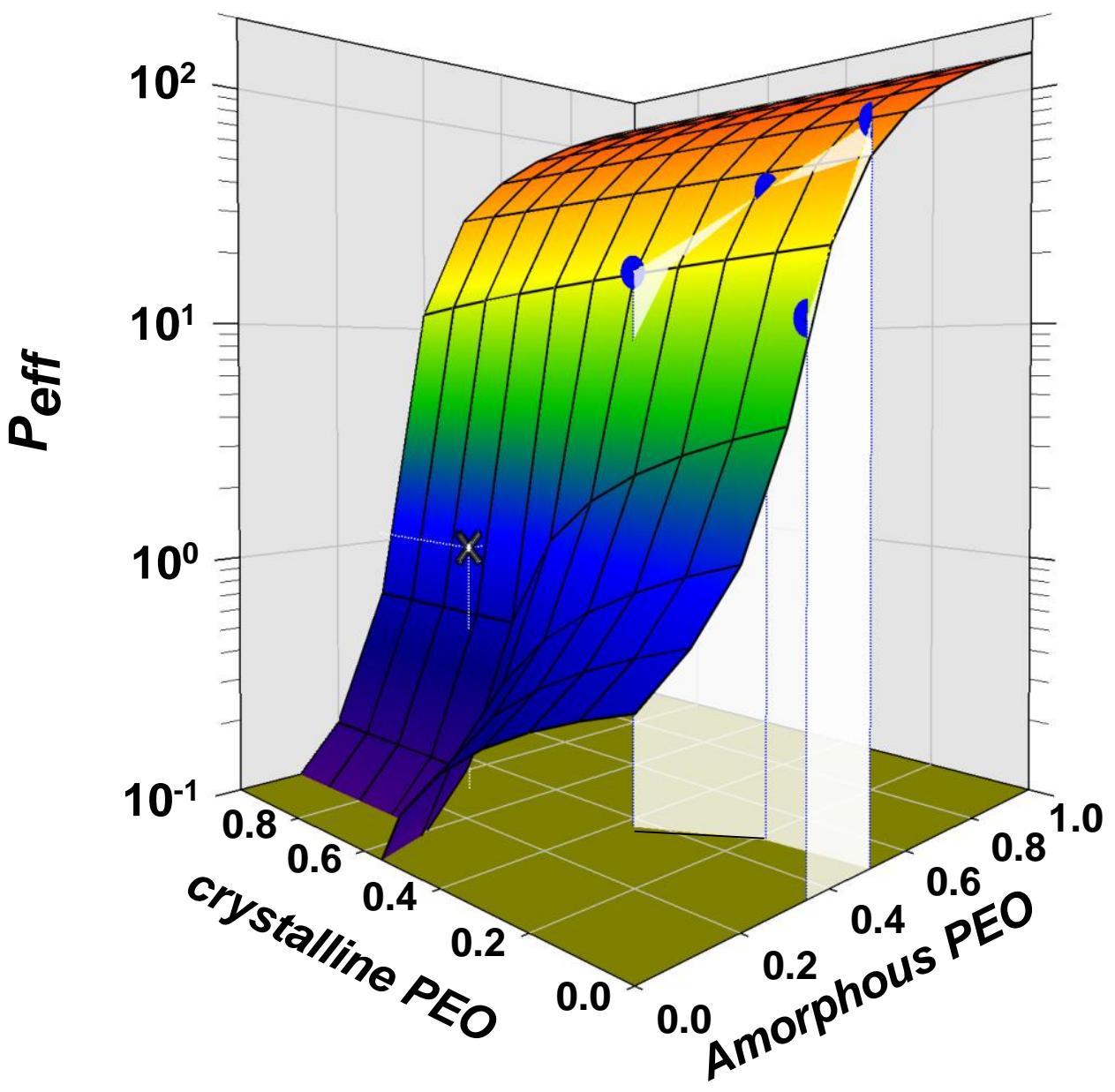

Figure 10.- Effective permeability of $\mathrm{CO}_{2}$ for a three-phase system of crystalline and amorphous PEO in a BKDA-ODA matrix and the predictions of EMA. 